\begin{tabular}{|c|c|c|}
\hline MATHRES & $\begin{array}{l}\text { Journal of Nonlinear Functional Analysis } \\
\text { Available online at http://jnfa.mathres.org }\end{array}$ & $\equiv \equiv$ \\
\hline
\end{tabular}

\title{
SOME KRASNOSEL'SKII TYPE RANDOM FIXED POINT THEOREMS
}

\author{
JOHN R. GRAEF ${ }^{1, *}$, JOHNNY HENDERSON $^{2}$, ABDELGHANI OUAHAB $^{3}$ \\ ${ }^{1}$ Department of Mathematics, University of Tennessee at Chattanooga, Chattanooga, TN 37403-2504, USA \\ ${ }^{2}$ Department of Mathematics, Baylor University, Waco, Texas 76798-7328, USA \\ ${ }^{3}$ Laboratory of Mathematics, Sidi-Bel-Abbès University, PoBox 89, 22000 Sidi-Bel-Abbès, Algeria
}

\begin{abstract}
The authors prove some random fixed point theorems for the sum of a weakly-strongly continuous random operator and an expansive random operator in Banach spaces. They also establish a random multivalued version of a Krasnosel'skii type fixed point theorem for the sum $B+G$, where $B$ is a linear or nonlinear random operator and $G$ is a random multivalued operator. They apply their results to a random fractional integral inclusion. A result for systems is also given. Their results extend previous ones in the literature.
\end{abstract}

Keywords. Multivalued map; Selection, Compact set; Fixed point; Random operator.

2010 Mathematics Subject Classification. 47H10, 47H40.

\section{Introduction}

Probabilistic functional analysis is an important mathematical discipline because of its applications to probabilistic models in applied problems. Random operator theory is needed for the study of various classes of random equations. However, it is usually the case that the mathematical models or equations used to describe phenomena in the biological, physical, engineering,

${ }^{*}$ Correspondign author.

E-mail addresses: John-Graef@utc.edu (J. R. Graef), Johnny_Henderson@baylor.edu (J. Henderson), agh_ouah ab@yahoo.fr (A. Ouahab).

Received July 24, 2017; Accepted September 13, 2017.

(C)2017 Journal of Nonlinear Functional Analysis 
and systems sciences contain certain parameters or coefficients that have specific interpretation$\mathrm{s}$, but whose values are unknown. Therefore, it is more realistic to consider such equations as random operator equations, which, in fact, are much more difficult to handle mathematically than deterministic equations. The problem of fixed points for random mappings was initialed by the Prague school of probabilists. The first results were obtained in 1955-1956 by S̆paček and Hans in the context of Fredholm integral equations with random kernels. In a separable metric space, random fixed point theorems for contraction mappings were proved by Hanš [1], Mukherjea [2], and S̆paček [3]. Random fixed point theorems of Schauder or Krasnosel'skii type were given by Bharucha-Reid [4], and Prakasa Rao [5]. In [6, 7], Itoh extended several well known fixed point theorems (that is, for contraction, non-expansive, and condensing mappings) to multivalued contraction mappings. Afterwards, various stochastic aspects of Schauder's, Covitz and Nadler's, Bohnenblust-Karlin's, and Kakutani-Ky Fan's fixed point theorems have been studied by Lin [8], Papageorgiou [9], Sehgal and Singh [10], and many other authors. Now it has become a full fledged research area, and a vast amount of mathematical activity has been carried out in this direction; see, for example, $[11,12]$ and the references therein.

In 1958, Krasnosel'skii [13] established that the equation

$$
A u+B u=u, \quad u \in M,
$$

has a solution in $M$ where $A$ and $B$ satisfy:

(i) $A x+B y \in M$ for all $x, y \in M$.

(ii) $A$ is continuous on $M$ and $\overline{A(M)}$ is a compact set in $X$.

(iii) $B$ is a $k$-contraction on $X$.

That result combined Banach's contraction principle and Schauder's fixed point theorem. The existence of fixed points for the sum of two deterministic operators has attracted tremendous interest, and their applications are frequent in nonlinear analysis. Many improvements of Krasnosel'skii's theorem have been established in the literature over the course of time by modifying the above assumptions; see, for example, [14, 15] and the references therein. Recently, Rao [5] obtained a probabilistic version of the Krasnosel'skii's theorem that involves the sum of a contraction random operator and a compact random operator on a closed convex subset of a separable Banach space. Itoh [7] extended Rao's result to a sum of a non-expansive random operator and a completely continuous random operator on a weakly compact convex subset of a 
separable uniformly convex Banach space. Very recently, Arunchai and Plubtieng [16] obtained a random fixed point theorem for the sum of a weakly-strongly continuous random operator and a non-expansive random operator that contains a Krasnosel'skii type fixed point theorem as a special case. Fixed point theory for multivalued mappings is an important topic in set-valued analysis. Several well-known fixed point theorems for single-valued mappings such as those of Banach and Schauder have been extended to multivalued mappings in Banach spaces; see $[17,18]$ and the references therein.

Our goal in this work is to give random versions of single and multivalued Krasnosel'skii type fixed point theorems. This paper is organized as follows. In Section 2, we introduce all the background material needed in this paper such as multivalued analysis and some fixed point theorems. Section 3 is devoted to establishing a random version of Krasnosel'skii's fixed point theorem for weakly-strongly continuous functions perturbed by an expansive operator. In Section 4, using an approximation method, we discuss the existence of solutions to equation (1.1) where $B$ is a random linear operator and $G$ is a random multivalued map. In Section 5, we apply our results to a random fractional integral inclusion. The aim of Section 6 is to present some random fixed point results for systems of random operators. In the final section of the paper, Section 7, we prove a random fixed point theorem in vector measure spaces.

\section{Preliminaries}

In this section, we recall from the literature some notations, definitions, and auxiliary results that will be used throughout this paper.

Let $(E,|\cdot|)$ be a Banach space. Denote by $\mathscr{P}(E)=\{Y \subset E: Y \neq \emptyset\}, \mathscr{P}_{c l}(E)=\{Y \in$ $\mathscr{P}(E): Y$ closed $\}, \mathscr{P}_{b}(E)=\{Y \in \mathscr{P}(E): Y$ bounded $\}, \mathscr{P}_{c v}(E)=\{Y \in \mathscr{P}(E): Y$ convex $\}$, $\mathscr{P}_{c p}(E)=\{Y \in \mathscr{P}(E): Y$ compact $\}$, and $\mathscr{P}_{w k c p}(E)=\{Y \in \mathscr{P}(E): Y$ weakly compact $\}$. Let $(X, d)$ and $(Y, \rho)$ be two metric spaces and $G: X \rightarrow \mathscr{P}_{c l}(Y)$ be a multi-valued map. A singlevalued map $g: X \rightarrow Y$ is said to be a selection of $G$, and we write $g \subset G$, if $g(x) \in G(x)$ for every $x \in X$. A mapping $G$ is called upper semi-continuous (u.s.c. for short) on $X$ if for each $x_{0} \in X$ the set $G\left(x_{0}\right)$ is a nonempty subset of $Y$, and if for each open subset $N$ of $Y$ containing $G\left(x_{0}\right)$, there exists an open neighborhood $M$ of $x_{0}$ such that $G(M) \subseteq N$. That is, if the set $G^{-1}(V)=\{x \in X, G(x) \cap V \neq \emptyset\}$ is closed for any closed set $V$ in $Y$. Equivalently, $G$ is u.s.c. if 
the set $G^{+1}(V)=\{x \in X, G(x) \subset V\}$ is open for any open set $V$ in $Y$. The mapping $G$ is lower semi-continuous (l.s.c.) if the set $\{x \in X: G(x) \cap V \neq \emptyset\}$ is open for any open set $V$ in $Y$.

Assume now that $X$ and $Y$ are normed linear spaces. We consider them as locally convex topological Hausdorff spaces with their weak topologies $\sigma\left(X, X^{*}\right)$ and $\sigma\left(Y, Y^{*}\right)$, respectively, where $X^{*}$ denotes the dual space of $X$. The multifunction $F: X \rightarrow \mathscr{P}(Y)$ is weakly-weakly upper semicontinuous (w.-w.u.s.c.) on $X$ if for every weakly closed set $M \subseteq Y$ the set $F^{-1}(M)=\{x \in$ $X: F(x) \cap M \neq \emptyset\}$ is sequentially weakly closed in $X$. We say that $F: X \rightarrow \mathscr{P}(Y)$ is weaklystrongly upper semicontinuous (w.-s.u.s.c.) on $X$ if for every weakly closed set $M \subseteq Y$, the set $F^{-1}(M)$ is closed (with the norm topology) in $X$.

Let $X$ be a normed space and $A \in \mathscr{P}(X)$. We denote the support function by

$$
\sigma\left(x^{*}, A\right)=\sup _{a \in A}\left(x^{*}, a\right), x^{*} \in X^{*} .
$$

Theorem 2.1. ([19]) A multivalued map $F: X \rightarrow \mathscr{P}(Y)$ is w.-w.u.s.c. (or w.-s.u.s.c.) on $X$ if for every sequence $\left\{x_{n}\right\}$ in $X$ weakly converging (converging in the norm topology in $X$, resp.) to $x$ and every sequence $\left\{y_{n}\right\}$ of $Y$ with $y_{n} \in F(x)$ for $n \in \mathbb{N}$, there exists a subsequence of $\left\{y_{n}\right\}$ weakly converging (converging in the norm topology in $Y$ ) to some $y \in F(x)$. If, in addition, there is a weakly compact set $C \subset Y$ such that $F(x) \subset C$ for $x \in X$, then the above conditions are also necessary for $F$ to be w.-w.u.s.c. on $X$.

Consider the Hausdorff-Pompeiu metric $H_{d}: \mathscr{P}(X) \times \mathscr{P}(X) \longrightarrow \mathbb{R}^{+} \cup\{\infty\}$ defined by

$$
H_{d}(A, B)=\max \left\{\sup _{a \in A} d(a, B), \sup _{b \in B} d(A, b)\right\},
$$

where $d(A, b)=\inf _{a \in A} d(a, b)$ and $d(a, B)=\inf _{b \in B} d(a, b)$. Then $\left(\mathscr{P}_{b, c l}(X), H_{d}\right)$ is a metric space and $\left(\mathscr{P}_{c l}(X), H_{d}\right)$ is a generalized metric space (see [19]).

Definition 2.1. A multivalued operator $N: X \rightarrow \mathscr{P}(X)$ is called:

(a) $\gamma$-Lipschitz if there exists $\gamma>0$ such that

$$
H_{d}(N(x), N(y)) \leq \gamma d(x, y), \text { for each } x, y \in X
$$

(b) a contraction if it is $\gamma$-Lipschitz with $\gamma<1$.

Let $(\Omega, \Sigma, \mu)$ be a complete $\sigma$-finite measurable space and $F: \Omega \rightarrow \mathscr{P}(X)$ be a multivalued mapping. Then $F$ is measurable if $F^{+1}(Q)=\{\omega \in \Omega: F(\omega) \subset Q\}$ for every $Q \in \mathscr{P}_{c l}(X)$. If $X$ 
is a metric space, we let $B(X)$ denote the Borel $\sigma$-algebra on $X$. Then the product $\sigma$-algebra on $\Omega \times X$, denoted by $\Sigma \otimes B(X)$, is the smallest $\sigma$-algebra on $\Omega \times X$ that contains all the sets $A \times S$ where $A \in \Sigma$ and $S \in B(X)$.

Definition 2.2. A mapping $F: \Omega \times X \rightarrow \mathscr{P}(X)$ is said to be a random operator if, for any $x \in X$, $F(\cdot, x)$ is measurable.

Definition 2.3. A random fixed point of $F$ is a measurable function $y: \Omega \rightarrow X$ such that $y(\omega) \in$ $F(\omega, y(\omega))$ for all $\omega \in \Omega$. Equivalently, a random fixed point of $F$ is a measurable selection for the multivalued map Fix $F_{\omega}: \Omega \rightarrow \mathscr{P}(X)$ defined by

$$
\operatorname{Fix}_{\omega}(x)=\{x \in X: x \in F(\omega, x)\}
$$

Definition 2.4. A multifunction $F: \Omega \rightarrow \mathscr{P}(X)$ is said to be graph measurable if

$$
\mathscr{G} r(G)=\{(\omega, x) \in \Omega \times X: x \in G(\omega)\}
$$

is member of $\Sigma \otimes B(X)$.

The following two results are needed in this paper. The first one is the celebrated KuratowskiRyll-Nardzewski selection theorem.

Theorem 2.2. ([20]) Let $(\Omega, \Sigma)$ be a measurable space, $Y$ be a separable metric space, and $F: \Omega \rightarrow \mathscr{P}_{c l}(Y)$ be a measurable multivalued function. Then $F$ has a measurable selection.

Theorem 2.3. Let $(\Omega, \Sigma)$ be a measurable space, $Y$ be a separable metric space, and $F: \Omega \longrightarrow$ $\mathscr{P}_{c l}(Y)$ be a multivalued map that is measurable. Then $F$ is graph-measurable.

The following important selection theorem is due to Yankov, von Neumann, and Aumann $($ see $[19,21])$.

Theorem 2.4. If $G: \Omega \rightarrow \mathscr{P}_{c p}(X)$ is a multivalued map such that the graph $\mathscr{G}_{r}(G)$ of $G$ is measurable, then $G$ has a measurable selector, where

$$
\mathscr{G} r(G)=\{(\omega, x) \in \Omega \times X: x \in G(\omega)\}
$$

The next theorem is due to Kannan [22]. 
Theorem 2.5. Let $X$ be a separable Banach space and $F: \Omega \times X \rightarrow \mathscr{P}_{c p}(X)$ be a random operator such that for each $\omega \in \Omega, F(\omega, \cdot)$ is u.s.c.. Then $F$ is $\Sigma \otimes \mathscr{B}(X)$ measurable, where $\Sigma \otimes \mathscr{B}(X)$ is the product $\sigma$-algebra on $\Omega \times X$.

Theorem 2.6. ([23]) Let $X$ be a separable metric space with $\left\{x_{k}\right\}$ a countable dense subset of $X$ and $Y$ be a separable Banach space. Let $F: \Omega \times X \rightarrow \mathscr{P}_{c p, c v}(Y)$ be an upper semicontinuous Carathéodory multifunction. Then the mapping $G: \Omega \times X \rightarrow \mathscr{P}_{c l, c v}(Y)$ defined by

$$
G(\omega, x)=\bigcap_{n=1}^{\infty} \overline{c o}\left\{F\left(\omega, x_{k}\right): d\left(x_{k}, x\right)<1 / n\right\}
$$

satisfies the following conditions:

(1) For each $\omega \in \Omega$ and $x \in X, \emptyset \neq G(\omega, x) \subset F(\omega, x)$.

(2) For each $\omega \in \Omega, G(\omega,$.$) is u.s.c.$

(3) $G$ is $\mathscr{L} \otimes \mathscr{B}(X)$ measurable.

Definition 2.5. A random operator $T: \Omega \times X \rightarrow X$ is said to be continuous at $x_{0} \in X$ if $\lim _{n \rightarrow \infty}\left\|x_{n}-x_{0}\right\|=0$ implies $\lim _{n \rightarrow \infty}\left\|T\left(\omega, x_{n}\right)-T(\omega, x)\right\|=0$ a.s.

The following results are due to Hanš [1].

Theorem 2.7. Let $X$ be a real separable Banach space, $T: \Omega \times X \rightarrow X$ be a continuous random operator, and let $k(\omega)$ be a nonnegative real valued random variable such that $k(\omega)<1$ a.s. and

$$
\left\|T\left(\omega, x_{1}\right)-T\left(\omega, x_{2}\right)\right\| \leq k(\omega)\left\|x_{1}-x_{2}\right\| \text { for each } x_{1}, x_{2} \in X, \omega \in \Omega .
$$

Then there exists a random variable $y: \Omega \rightarrow X$ that is the unique fixed point of $T$.

Lemma 2.1. Let $X$ be a real separable Banach space, $T: \Omega \times X \rightarrow X$ be a continuous random operator, $k(\omega)$ be a nonnegative real valued random variable such that $k(\omega)<1$ a.s. Assume that for some integer $n \in \mathbb{N}$, we have

$$
\left\|T_{\omega}^{n}\left(x_{1}\right)-T_{\omega}^{n}\left(x_{2}\right)\right\| \leq k(\omega)\left\|x_{1}-x_{2}\right\| \text { for } x_{1}, x_{2} \in X
$$

where

$$
T_{\omega}(\cdot)=T(\omega, \cdot), T_{\omega}^{n}(\cdot)=T\left(\omega, T^{n-1}(\omega, \cdot)\right) .
$$

Then there exists a random variable $y: \Omega \rightarrow X$ that is the unique fixed point of $T$. 
Theorem 2.8. Let $T: \Omega \times X \rightarrow X$ be an almost surely continuous random operator. Assume that there exist a real-valued random variable $k$ such that

$$
\mu\left(\left\{\omega:\left\|T\left(\omega, x_{1}\right)-T\left(\omega, x_{2}\right)\right\| \leq k(\omega)\|x-y\|\right\}\right)=1
$$

Then for every real number $\lambda \neq 0$ such that $k(\omega)<|\lambda|$ and

$$
\mu\{\omega \in \Omega: k(\omega)<|\lambda|\}=1
$$

there exists a random operator $S$ that is the inverse of the random operator $\left(T(\omega, \cdot)-\lambda I_{X}\right)$, where $I_{X}$ denotes the identity operator.

Theorem 2.9. ([19]) Let $X$ be a separable metric space, $Y$ a metric space $f: \Omega \times X \rightarrow X$ be a Carathéodory function, and $U$ an open subset of $Y$. Then the multivalued map $F: \Omega \rightarrow \mathscr{P}(X)$ defined by $F(\omega)=\{\omega \in \Omega: f(t, x) \in U\}$ is measurable. In particular if $f$ is real valued, then

$$
F_{*}(\omega)=\{\omega \in \Omega: f(\omega, x)>\lambda\}, \quad \widetilde{F}(\omega)=\{\omega \in \Omega: f(\omega, x)<\lambda\}
$$

are measurable.

\section{Expansive mappings}

In this section, we obtain fixed point theorems for the sum of two operators, one of which is expansive.

Definition 3.1. Let $X$ be a normed space and let $M \subseteq X$. An operator $T: M \rightarrow X$ is said to be expansive on $M$, if there is a constant $h>1$ such that $\|T(x)-T(y)\| \geq h\|x-y\|$ for all $x, y \in M$.

We begin with a result of Xiang and Yuan [24].

Theorem 3.1. Let $X$ be a complete metric space and $M$ be a closed subset of $X$. If $B: M \rightarrow X$ is expansive and $M \subseteq B(M)$, then there exists a unique point $x \in M$ such that $x=B(x)$.

For our first theorem in this section, we will need the concept of a weakly-strongly continuous mapping. 
Definition 3.2. Let $X$ be a Banach space and $M$ be a closed subset of $X$. A mapping $T: M \rightarrow X$ is weakly-strongly continuous iffor each sequence $\left\{x_{n}\right\}$ in $M$ which converges weakly to $x \in M$, the sequence $\left\{T\left(x_{n}\right)\right\}$ converges strongly to $T(x)$.

In what follows we will need notion of an expansive random operator.

Definition 3.3. The random operator $T: \Omega \times X \rightarrow X$ is said be random expansive if there exists a positive real random variable $k>1$ such that

$$
\left\|T\left(\omega, x_{1}\right)-T\left(\omega, x_{2}\right)\right\| \geq k(\omega)\left\|x_{1}-x_{2}\right\| \text { for all } x_{1}, x_{2} \in X, \omega \in \Omega
$$

We will also need the following two lemmas.

Lemma 3.1. Let $X$ be a real separable Banach space and $M$ be a closed subset of $X$. Assume that the random operator $T: \Omega \times M \rightarrow X$ is continuous and random expansive. Then the inverse of $T(\omega, \cdot)-I_{X}: M \rightarrow\left(T(\omega, \cdot)-I_{X}\right)(M)$, for every $\omega \in \Omega$, exists and

$$
\begin{aligned}
\|\left(T(\omega, \cdot)-I_{X}\right)^{-1}\left(y_{1}\right)-( & \left.T(\omega, \cdot)-I_{X}\right)^{-1}\left(y_{2}\right) \| \\
\leq & \frac{1}{k(\omega)-1}\left\|y_{1}-y_{2}\right\| \text { for all } y_{1}, y_{2} \in\left(T(\omega, \cdot)-I_{X}\right)^{-1}(M) .
\end{aligned}
$$

Proof. Let $\omega \in \Omega$ and $x_{1}, x_{2} \in M$ be such that

$$
T\left(\omega, x_{1}\right)-x_{1}=T\left(\omega, x_{2}\right)-x_{2}
$$

Then,

$$
\left\|T\left(\omega, x_{1}\right)-x_{1}-T\left(\omega, x_{2}\right)+x_{2}\right\| \geq(k(\omega)-1)\left\|x_{1}-x_{2}\right\| \quad \text { implies } \quad x_{1}=x_{2},
$$

which shows that $T(\omega, \cdot)-I_{X}$ is one to one. Hence, the inverse of $T(\omega, \cdot)-I_{X}: M \rightarrow(T(\omega, \cdot)-$ $\left.I_{X}\right)(M)$ exists and (3.2) holds.

Lemma 3.2. Let $X$ be a separable Banach space, $M$ be a closed subset of $X$, and assume that the mapping $B: M \rightarrow X$ is expansive with constant $h>1$. Then $(I-B)(M)$ is closed.

Proof. Let $\left\{y_{n}: n \in \mathbb{N}\right\} \subset(I-B)(M)$ be a sequence converging to $y$. We will show that $y \in(I-B)(M)$. For every $n \in \mathbb{N}$ there exists $x_{n} \in M$ such that $y_{n}=(I-B)\left(x_{n}\right)$. By Lemma 3.1,

$$
\left\|x_{n}-x_{m}\right\|=\left\|(I-B)^{-1}\left(y_{n}\right)-(I-B)^{-1}\left(y_{m}\right)\right\| \leq \frac{1}{h-1}\left\|y_{n}-y_{m}\right\| \text { for } n, m \in \mathbb{N} .
$$


Hence, $\left\{x_{n}\right\}$ is Cauchy sequence in $X$. Thus, there exists $x \in X$ such that $\lim _{n \rightarrow \infty} x_{n}=x \in M$. Since $(I-B)^{-1}$ is continuous, we concluded that $(I-B)^{-1}\left(y_{n}\right) \rightarrow(I-B)^{-1}(y)$, as $n \rightarrow \infty$. Therefore, $y=(I-B)(x) \in(I-B)(M)$.

Theorem 3.2. Let $X$ be a Banach space, $M \subset X$ be a nonempty weakly compact convex set, and $B$ and $T$ be two maps from $M$ into $X$ such that:

(i) $B$ is expansive;

(ii) $T$ is weakly-strongly continuous;

(iii) For each $z \in T(M)$ we have $M \subseteq B(M)+z$, where

$$
T(M)+z=\{y+z: y \in T(M)\} .
$$

Then Fix $(B+T)=\{x \in M: x=B(x)+T(x)\}$ is nonempty and closed.

Proof. For fixed $y \in M$, we consider $F^{y}: M \rightarrow X$ defined by

$$
F^{y}(x)=B(x)+T(y), x \in M .
$$

Note that for each fixed $y \in M, F^{y}$ is expansive since $B$ is expansive, and from (iii) we have $M \subseteq F^{y}(M)$. Hence, by Theorem 3.1, there exists a unique fixed point $x(y) \in M$ such that

$$
F^{y}(x(y))=x(y)=B(x(y))+T(y) .
$$

Since $B$ is expansive, there exists $h>1$ such that $\left\|B\left(x\left(y_{1}\right)\right)-B\left(x\left(y_{2}\right)\right)\right\| \geq h\left\|x\left(y_{1}\right)-x\left(y_{2}\right)\right\|$. Then,

$$
\begin{aligned}
\left\|B\left(x\left(y_{1}\right)\right)-B\left(x\left(y_{2}\right)\right)\right\| & =\left\|x\left(y_{1}\right)-T\left(y_{1}\right)-\left(x\left(y_{2}\right)-T\left(y_{2}\right)\right)\right\| \\
& \leq\left\|x\left(y_{1}\right)-x\left(y_{2}\right)\right\|+\left\|T\left(y_{1}\right)-T\left(y_{2}\right)\right\| .
\end{aligned}
$$

So

$$
\left\|x\left(y_{1}\right)-x\left(y_{2}\right)\right\|+\left\|T\left(y_{1}\right)-T\left(y_{2}\right)\right\| \geq h\left\|x\left(y_{1}\right)-x\left(y_{2}\right)\right\|,
$$

or

$$
\left\|x\left(y_{1}\right)-x\left(y_{2}\right)\right\| \leq \frac{1}{h-1}\left\|T\left(y_{1}\right)-T\left(y_{2}\right)\right\| .
$$

To show that $x(\cdot): M \rightarrow M$ is continuous, let $\left\{y_{n}: n \in \mathbb{N}\right\} \subseteq M$ be a sequence converging weakly to $y$. Since $T$ is weakly-strongly continuous, $\left\|T\left(y_{n}\right)-T(y)\right\| \rightarrow 0$ as $n \rightarrow \infty$. This implies $\left\|x\left(y_{n}\right)-x(y)\right\| \leq \frac{1}{h-1}\left\|T\left(y_{n}\right)-T(y)\right\| \rightarrow 0$ as $n \rightarrow \infty$. Hence, $x(\cdot)$ is continuous. 
Let $L: M \rightarrow M$ be defined by $L(z)=x(T(z))$. It is clear that $L$ is weakly continuous, and by Schauder's fixed point theorem, there exists $z \in M$ such that $x(T(z))=z$. Hence,

$$
B(z)+T(z)=z
$$

and so the set Fix $(B+T)$ is nonempty.

To show that $F i x(B+T)$ is closed, let $\left\{x_{n}: n \in \mathbb{N}\right\} \subset F i x(B+T)$ be a sequence converging to $x$. We will show that $x \in F i x(B+T)$. For each $n \in \mathbb{N}$, we have $x_{n}=B\left(x_{n}\right)+T\left(x_{n}\right)$, so $(I-B)\left(x_{n}\right)=T\left(x_{n}\right) \in(I-B)(M)$. From Lemma 3.1, we have

$$
x_{n}=(I-B)^{-1}\left(T\left(x_{n}\right)\right), \quad n \in \mathbb{N} .
$$

Since $T$ is weakly-strongly continuous, $\left\{T\left(x_{n}\right)\right\}$ converge strongly to $T(x)$. By Lemma 3.1, we have

$$
\left\|(I-B)^{-1}\left(T\left(x_{n}\right)\right)-(I-B)^{-1}(T(x))\right\| \leq \frac{1}{h-1}\left\|T\left(x_{n}\right)-T(x)\right\| \rightarrow 0 \quad \text { as } n \rightarrow \infty .
$$

Therefore,

$$
x=(I-B)^{-1}(T(x)) \text { implies } \quad x=B(x)+T(x) .
$$

Hence, Fix $(B+T)$ is closed, and this completes the proof of the theorem.

Theorem 3.3. Let $X$ be a real separable Banach space and $M$ be a closed subset of X. Assume that the random operator $T: \Omega \times X \rightarrow X$ is continuous, random expansive, and

$$
M \subset T(\omega, M), \text { for every } \omega \in \Omega
$$

Then there exists a random variable $y: \Omega \rightarrow M$ that is the unique fixed point of $T$.

Proof. Let $\omega \in \Omega$ and $T(\omega, \cdot): M \rightarrow T_{\omega}(M)$. From (3.1), the inverse of $T_{\omega}, T_{\omega}^{-1}: T_{\omega}(M) \rightarrow M$, exists. Let $x_{1}, x_{2}, y_{1}, y_{2} \in M$ be such that

$$
T\left(\omega, x_{1}\right)=y_{1}, T\left(\omega, x_{2}\right)=y_{2} .
$$

Then,

$$
\left\|y_{1}-y_{2}\right\| \geq k(\omega)\left\|x_{1}-x_{2}\right\| \quad \text { implies } \quad\left\|T_{\omega}^{-1}\left(y_{1}\right)-T_{\omega}^{-1}\left(y_{2}\right)\right\| \leq \frac{1}{k(\omega)}\left\|y_{1}-y_{2}\right\| .
$$

By Banach's fixed point theorem, there exists a unique $y_{\omega} \in M$ that is a fixed point of $T_{\omega}^{-1}$. That is, $T_{\omega}^{-1}\left(y_{\omega}\right)=y_{\omega}$, and so $y_{\omega}=T_{\omega}\left(y_{\omega}\right), \omega \in \Omega$. 
Define a multivalued mapping $G: \Omega \rightarrow \mathscr{P}(M)$ by

$$
G(\omega)=\{y \in M: y=T(\omega, y)\}
$$

Since $T(\omega, \cdot)$ is a continuous mapping, for every $\omega \in \Omega$, we have $G(\omega) \in \mathscr{P}_{c p}(M)$. We define $G_{n}: \Omega \rightarrow \mathscr{P}_{c l}(M)$ by

$$
\begin{aligned}
G_{n}(\omega) & =\left\{\omega \in \Omega:\|y-T(\omega, y)\|<\frac{1}{n}\right\} \\
& =\left\{\omega \in \Omega: y-T(\omega, y) \in B\left(0, \frac{1}{n}\right)\right\} .
\end{aligned}
$$

Since $(\omega, y) \rightarrow y-T(\omega, y)$ is a Carathéodory function, from Theorem 2.9, the set multivalued map $G_{n}$ is measurable, so

$$
\overline{G_{n}(\omega)}=\overline{\left\{\omega \in \Omega: y-T(\omega, y) \in B\left(0, \frac{1}{n}\right)\right\}}
$$

is measurable. Moreover,

$$
G(\omega)=\bigcap_{n=1}^{\infty} \overline{G_{n}(\omega)}, \quad \omega \in \Omega .
$$

From Theorem 2.2, there exists a measurable selection $y: \Omega \rightarrow M$ of $G$ that in turn is a unique random fixed point of $T$.

We can easily prove the following lemma.

Lemma 3.3. Let $X$ be a real separable Banach space and $T: \Omega \times X \rightarrow X$ be a continuous random operator. Let $k(\omega)$ be a nonnegative real valued random variable such that $k(\omega)>1$ a.s., and assume that for some integer $n \in \mathbb{N}$ and all $\omega \in \Omega$, we have

$$
\left\|T_{\omega}^{n}\left(x_{1}\right)-T_{\omega}^{n}\left(x_{2}\right)\right\| \geq k(\omega)\left\|x_{1}-x_{2}\right\| \text { for all } x_{1}, x_{2} \in X
$$

Then there exists a random variable $y: \Omega \rightarrow X$ that is the unique fixed point of $T$.

Our next theorem guarantees the existence of a fixed point for the sum of a random operator and an expansive random operator.

Theorem 3.4. Let $X$ be a separable Banach space and $M \subset X$ be a nonempty compact convex set. Assume that $T, B: \Omega \times M \rightarrow X$ are random operators such that:

$\left(\mathscr{A}_{1}\right) T$ is a continuous random operator;

$\left(\mathscr{A}_{2}\right)$ B is a continuous random expansive operator;

$\left(\mathscr{A}_{3}\right) T(\omega, M) \subseteq(I-B(\omega, \cdot))(M), \omega \in \Omega$. 
Then $B+T$ has at least one random fixed point.

Proof. Let $\omega \in \Omega, y \in M$, and $F_{\omega, y}: M \rightarrow M$ be defined by

$$
F_{\omega, y}(x)=B(\omega, x)+T(\omega, y) .
$$

As before, since $B$ is expansive, $F_{\omega, y}$ is expansive for each fixed $\omega$ and $y$. So by Theorem 3.1, there exists a unique fixed point of $F_{\omega, y}(\cdot)$, and by Lemma 3.1, $(I-B(\omega, \cdot))^{-1}$ exists. We define the operator $N_{\omega}: M \rightarrow M$ by $N_{\omega}(y)=(I-B(\omega, \cdot))^{-1} T(\omega, y)$. It is easy to see that by Schauder's fixed point theorem, $N_{\omega}$ has at least one fixed point. Define a mapping $S: \Omega \rightarrow \mathscr{P}(M)$ by

$$
S(\omega)=\{y \in M: y=B(\omega, y)+T(\omega, y)\}
$$

Since $B(\omega, \cdot)+T(\omega, \cdot)$ is a continuous mapping, for every $\omega \in \Omega$, we have $S(\omega) \in \mathscr{P}_{c p}(M)$. As in the proof of Theorem 3.3, we can prove that $S$ is measurable. From Theorem 2.2, there exists a measurable selection $y: \Omega \rightarrow M$ of $S$ that is a random fixed point of $B+T$. This completes the proof of the theorem.

Since a weakly-strongly continuous random operator is continuous, we immediately have the following result.

Theorem 3.5. Let $X$ be a separable Banach space and $M$ be a nonempty compact convex subset of $X$. Assume that T, $B: \Omega \times M \rightarrow X$ are random operators such that:

$\left(\mathscr{A}_{4}\right) T$ is a weakly-strongly continuous random operator;

$\left(\mathscr{A}_{5}\right) B$ is a continuous random expansive operator;

(A) For each $y \in M$, we have

$$
M \subseteq B(\omega, M)+T(\omega, y), \omega \in \Omega
$$

Then $B+T$ has at least one random fixed point.

\section{Approximation method and Krasnosel'skii-type fixed point theorems}

For a fairly general class of multifunctions with compact and nonconvex values, and using a different method, approximate continuous selections have been constructed by Cellina [25], Górniewicz, Granas, and Kryszewski [26], and Górniewicz and Lassonde [27]. They have been 
used to develop an index theory. In this section, we use a technique of approximation proved recently for a version of a Krasnosel'skii-type fixed point result. The following continuous approximation theorem plays a key role in this section.

Theorem 4.1. ([19]) Let $(X,|\cdot|)$ be a normed space, $(Y,\|\cdot\|)$ be a Banach space, and $F: X \rightarrow$ $\mathscr{P}_{c v}(Y)$ be an u.s.c. multivalued map. Then, for every $\varepsilon>0$, there exists a locally Lipschitzian function $f_{\varepsilon}: X \rightarrow Y$ such that

$$
f_{\varepsilon}(X) \subseteq \operatorname{coF}(X)
$$

and

$$
\mathscr{G} r\left(f_{\varepsilon}\right) \subseteq \mathscr{G} r(F)+\varepsilon B_{*},
$$

where $B_{*}$ is an open unit ball in $X \times Y$.

Our first result in this section is the following.

Theorem 4.2. Let $X$ be a separable Banach space, $M$ be a compact convex subset of $X, G$ : $\Omega \times M \rightarrow \mathscr{P}_{c p, c v}(X)$ be an random multivalued map, and $B: \Omega \times M \rightarrow M$ be a continuous random operator. Assume that $G$ and $B$ satisfy the following conditions:

$\left(\mathscr{H}_{1}\right) G(\omega, \cdot)$ is a u.s.c. multivalued mapping for every $\omega \in \Omega$, and $G(\cdot, x)$ is measurable;

$\left(\mathscr{H}_{2}\right) B(\omega, \cdot)$ is a continuous expansive operator for every $\omega \in \Omega$;

$\left(\mathscr{H}_{3}\right) G(\omega, M) \subseteq(I-B(\omega, \cdot))(M), \omega \in \Omega$.

Then there exist a random operator $y: \Omega \rightarrow M$ such that

$$
y(\omega) \in B(\omega, y(\omega))+G(\omega, y(\omega)), \omega \in \Omega .
$$

Proof. By Theorem 2.6, there exists a multivalued jointly measurable u.s.c. map $G_{*}: \Omega \times M \rightarrow$ $\mathscr{P}_{c p, c v}(X)$ such that

$$
G_{*}(\omega, x) \subset G(\omega, x), \quad \omega \in \Omega, x \in M
$$

Let $\omega \in \Omega$; by Theorem 4.1, given $\varepsilon>0$, there exists a locally Lipschitzian map $f_{\varepsilon}(\omega, \cdot): M \rightarrow$ $X$ such that

$$
\mathscr{G}_{r}\left(f_{\varepsilon}(\omega, \cdot)\right) \subset \mathscr{G}_{r} G_{*}(\omega, .)+\varepsilon B_{*}
$$

and

$$
f_{\varepsilon}(\omega, M) \subset \operatorname{co} G_{*}(\omega, M)
$$


From $\left(\mathscr{H}_{3}\right)$, for every $y \in M$, we have $f(\omega, M) \subseteq(I-B(\omega, \cdot))(M)$. Hence for every $x \in M$ there exists $y \in M$ such that $y-B(\omega, y)=f(\omega, x)$. From Lemma 3.1, we have

$$
y=(I-B(\omega, \cdot))^{-1} f_{\varepsilon}(\omega, x)
$$

Define the operator $N_{\varepsilon}(\omega, \cdot): M \rightarrow M$ by

$$
N_{\varepsilon}(\omega, x)=(I-B)^{-1} f_{\varepsilon}(\omega, x)
$$

It easy to see that $N_{\varepsilon}$ satisfies the conditions of Schauder's fixed point theorem, and so there exists $x_{\varepsilon}(\omega, \cdot) \in M$ such that

$$
x_{\varepsilon}(\omega, \cdot)=B\left(\omega, x_{\varepsilon}(\omega, \cdot)\right)+f_{\varepsilon}\left(\omega, x_{\varepsilon}(\omega, \cdot)\right) \text {. }
$$

Let $\left\{\varepsilon_{n}: n \in \mathbb{N}\right\}$ be such that $\varepsilon_{n} \rightarrow 0$ as $n \rightarrow \infty$, and construct a sequence $\left\{x_{\varepsilon_{n}}(\omega): n \in \mathbb{N}\right\}$ in the above manor. Since $M$ is compact, there exists a subsequence of $x_{\varepsilon_{n}}(\omega)$, which we again denote by $x_{\varepsilon_{n}}(\omega)$, converging to $x(\omega) \in M$. So

$$
f_{\varepsilon_{n}}\left(\omega, x_{\varepsilon_{n}}(\omega)\right)=(I-B)\left(\omega, x_{\varepsilon_{n}}(\omega)\right) \rightarrow(I-B)(\omega, x(\omega)) \text { as } n \rightarrow \infty
$$

From (4.1), we obtain

$$
d\left(\left(x_{\varepsilon_{n}}(\omega), f_{\varepsilon_{n}}\left(\omega, x_{\varepsilon_{n}}(\omega)\right)\right), \mathscr{G}_{r}\left(G_{*}(\omega, \cdot)\right)\right) \leq \varepsilon_{n}, \text { for every } n \in \mathbb{N} .
$$

It is clear that $G(\omega, \cdot)$ has a closed graph in $X \times X$ and consequently $(I-B)(\omega, x) \in G_{*}(\omega, x)$. This implies that $x(\omega) \in B(\omega, x(\omega))+G_{*}(\omega, x(\omega))$. We define the multivalued operator $F$ : $\Omega \rightarrow \mathscr{P}_{c l}(M)$ by

$$
F(\omega)=\left\{x \in M: x \in B(\omega, x)+G_{*}(\omega, x)\right\} .
$$

Let $\phi: \Omega \times M \rightarrow \mathscr{P}_{c l}\left(\mathbb{R}_{+}\right)$be defined by $\phi(\omega, x)=d\left(x, B(\omega, x)+G_{*}(\omega, x)\right)=(d \circ H)(\omega, x)$, where $H: \Omega \times M \rightarrow M \times M$ is given by

$$
H(\omega, x)=\left(x, B(\omega, x)+G_{*}(\omega, x)\right), \quad(\omega, x) \in \Omega \times M .
$$

is a jointly measurable multifunction. Then $\phi(\cdot, \cdot)$ is measurable. Hence,

$$
\begin{aligned}
\mathscr{G} r(F) & =\{(\omega, x) \in \Omega \times M: x \in F(\omega)\} \\
& =\{(\omega, x) \in \Omega \times M: d(x, \phi(\omega, x))=0\} \\
& =\phi^{-1}(\{0\}) .
\end{aligned}
$$


is measurable. By Theorem 2.4, $F$ has a measurable selection $y: \Omega \rightarrow M$ that is a random fixed point of $B+G$.

We can easily prove the following result.

Theorem 4.3. Let $X$ be a separable Banach space and $M$ be a closed bounded convex subset of $X$. Assume that $G: \Omega \times M \rightarrow \mathscr{P}_{c p, c v}(X)$ is a random multivalued map such that for each $\omega \in \Omega, G(\omega, \cdot)$ is u.s.c., B satisfies $\left(\mathscr{H}_{2}\right)-\left(\mathscr{H}_{3}\right)$, and for $\omega \in \Omega$,

$\left(\mathscr{H}_{4}\right) G(\omega, M)$ is compact.

Then the operator $B+G$ has at least one fixed point.

Proof. For fixed $\omega \in \Omega$, we have $N_{\varepsilon}(\omega, M) \subseteq M$, where $N_{\varepsilon}$ is defined in (4.2). By ( $\left.\mathscr{H}_{3}\right)$ we have

$$
N_{\varepsilon}(\omega, M)=(I-B(\omega, \cdot))^{-1} f_{\varepsilon}(\omega, M) \subseteq(I-B(\omega, .))^{-1}(G(\omega, M)) .
$$

From $\left(\mathscr{H}_{4}\right)$, we concluded that $N_{\varepsilon}(\omega, M)$ is compact. By Schauder's fixed point theorem, there exists $x_{\varepsilon}(\omega) \in M$ such that

$$
x_{\varepsilon}(\omega)=B\left(\omega, x_{\varepsilon}(\omega)\right)+f_{\varepsilon}\left(\omega, x_{\varepsilon}(\omega)\right) .
$$

By the same method used in the proof of Theorem 4.2, we can show that there exists a random function $y: \Omega \rightarrow M$ that is a fixed point of $B+G$.

In our next result we ask that $B$ be is a 1-coercive operator as given in the following definition.

Definition 4.1. Let $X$ be a normed space and let $M \subseteq X$. An operator $T: M \rightarrow X$ is said to be 1-coercive on $M$ if $\|T(x)-T(y)\| \geq\|x-y\|$ for all $x, y \in M$.

Theorem 4.4. Let $X$ be a separable Banach space and $M$ be a compact convex subset of X. Let $B: \Omega \times M \rightarrow X$ be a random continuous 1-coercive operator and $G: \Omega \times M \rightarrow \mathscr{P}_{c p, c v}(X)$ be a random multivalued map such that $G(\omega, \cdot)$ is u.s.c. Assume that

$$
\left(\mathscr{H}_{\alpha}\right) \quad \alpha>1, G(\omega, M) \subseteq(I-\alpha B(\omega, \cdot))(M) .
$$

Then $B+G$ has at least one random fixed point.

Proof. Fix $\omega \in \Omega$, and for each $n \in \mathbb{N}$, let $\alpha_{n}>1$ with $\alpha_{n} \rightarrow 1$ as $n \rightarrow \infty$. Then $\alpha_{n} B(\omega, \cdot)$ is an expansive operator. From Theorem 4.2, for each $n \in \mathbb{N}$, there exists $x_{n} \in M$ such that

$$
x_{n} \in \alpha_{n} B\left(\omega, x_{n}\right)+G\left(\omega, x_{n}\right) \quad \text { implies } \quad x_{n} \in B\left(\omega, \alpha_{n} x_{n}\right)+G\left(\omega, x_{n}\right) .
$$


Since $M$ is compact, there exists a subsequence of $\left\{x_{n}\right\}$ converging to $x \in M$. Let $L_{\omega}:[0,1] \times$ $M \rightarrow \mathscr{P}_{c p}(X)$ be a multivalued operator defined by

$$
(\alpha, x) \rightarrow L_{\omega}(\alpha, x)=\alpha B(\omega, x)+G(\omega, x)
$$

Using the fact that $B(\omega, \cdot)$ is a continuous operator, $G(\omega, \cdot)$ is $u . s . c$. , and $M$ is compact, we know that $L_{\omega}$ has a closed graph, and so

$$
x_{n} \in \alpha_{n} B\left(\omega, x_{n}\right)+G\left(\omega, x_{n}\right) \rightarrow x \in B(\omega, x)+G(\omega, x) \text { as } n \rightarrow \infty .
$$

Hence, $B+G$ has at least one random fixed point.

The final theorem in this section is our global multivalued version of the Krasonsel'skii fixed point theorem.

Theorem 4.5. Let $X$ be a separable Banach space and $G: \Omega \times X \rightarrow \mathscr{P}_{c p, c v}(X)$ be an multivalued map such that $\omega \rightarrow G(\omega, \cdot)$ is u.s.c. and $x \rightarrow G(\cdot, x)$ is measurable. Assume condition $\left(\mathscr{H}_{2}\right)$ holds and $G$ is compact. Then, either

(a) $x(\omega) \in \lambda B\left(\omega, \frac{x}{\lambda}\right)+\lambda G(\omega, x)$ has a random solution for $\lambda=1$,

or

(b) the set

$$
\left\{x: \Omega \rightarrow X \text { is a random variable } \mid x(\omega) \in \lambda B\left(\omega, \frac{x(\omega)}{\lambda}\right)+\lambda G(\omega, x(\omega)), \lambda \in(0,1), \omega \in \Omega\right\}
$$

is unbounded.

\section{Random fractional integral inclusions}

Random differential equations and random integral equations have been studied systematically in Ladde and Lakshmikantham [28]. Fractional differential equations arise quite naturally in various fields such as rheology, fractals, chaotic dynamics, modeling, control theory, signal processing, bioengineering, and biomedicine. They are useful to describe memory and hereditary properties of various materials and processes. Very recently, fractional differential equation$\mathrm{S}$ with random parameters have been studied by Lupulescu and Ntouyas [29] and Lupulescu, O’Regan and G. Rahman [30]. 
In this section, we present some applications of our fixed point results to the integral fractional inclusion

$$
x(\omega, t) \in x_{0}(\omega, t)+\frac{1}{\Gamma(\alpha)} \int_{0}^{t}(t-s)^{\alpha-1} F(\omega, s, x(\omega, s)) d s+p(\omega, t, x(\omega, t)), \quad t \in J,
$$

where $J:=[0, b], \alpha, \in(0,1), \Gamma(\cdot)$ denotes the gamma function, $F: \Omega \times J \times \mathbb{R} \rightarrow \mathscr{P}_{c v, c p}(\mathbb{R})$ is a random multivalued map, and $x_{0}: \Omega \rightarrow C(J, \mathbb{R})$ is a random variable for which we denote the $\operatorname{map} x_{0}(\omega)(t): J \rightarrow \mathbb{R}$ by $x_{0}(\omega, t)$.

Observe that the above inclusion can be written as $x(\omega, t) \in G(\omega, x(\omega, t))+B(\omega, x(\omega, t))$, where $B: \Omega \times \mathbb{R} \rightarrow \mathbb{R}$ is the random superposition operator defined by

$$
B(\omega)(x(\cdot))=B(\omega, x(\cdot)):=x_{0}(\omega, \cdot)+p(\omega, \cdot x(\cdot)), \omega \in \Omega, x \in C(J, \mathbb{R}),
$$

$G$ is the Volterra multivalued random integral operator of fractional order

$$
G(\omega, x)=\left\{h \in C(J, \mathbb{R}): h(t)=\frac{1}{\Gamma(\alpha)} \int_{0}^{t}(t-s)^{\alpha-1} f(s) d s: f \in S_{F, \omega, x}\right\},
$$

and

$$
S_{F, \omega, x}=\{f \in L(J, \mathbb{R}): f(t) \in F(\omega, t, x)\} .
$$

If $X$ and $Y$ are two locally compact metric spaces, then by $C(X, Y)$ we mean the space of continuous functions from $X$ into $Y$ endowed with the compact-open topology.

Lemma 5.1. ([9]) The function $f: \Omega \times X \rightarrow Y$ is Carathéodory if and only if the mapping $\omega \rightarrow r(\omega)(\cdot)=f(\omega, \cdot)$ is a measurable function from $\Omega \rightarrow C(X, Y)$.

On $[0, b]$, we consider the Lebesgue measure $\lambda(\cdot)$ and the $\sigma$-field, $\Sigma_{b}$, of Lebesgue measurable sets.

Lemma 5.2. Let $(\Omega, \Sigma, \mu)$ be a complete $\sigma$-finite measurable space, $X$ be a separable Banach space, and $F: \Omega \times[0, b] \times X \rightarrow \mathscr{P}_{c p, c v}(X)$ be a multivalued map. Assume that:

$\left(\mathscr{I}_{1}\right)$ For all $(\omega, t) \in \Omega \times[0, b], F(\omega, t, \cdot)$ is u.s.c., and for all $x \in \mathbb{R}, F(\cdot, \cdot, x)$ is jointly measurable.

$\left(\mathscr{I}_{2}\right)$ For all $x \in X$, we have $\|F(\omega, t, x)\|_{\mathscr{P}}=\sup \{|v|: v(t) \in F(\omega, t, x(t))\} \leq \gamma_{*}(\omega, t), \quad$ a.e. $t$ and for all $\omega \in \Omega$, where $\gamma_{*}: \Omega \times[0, b] \rightarrow X$ is a jointly measurable function that is continuous in $t$. 
Then the multifunction $G: \Omega \times C([0, b], X) \rightarrow \mathscr{P}(C([0, b], X))$ defined by

$$
G(\omega, x):=\left\{h \in C([0, b], X): h(t)=\frac{1}{\Gamma(\alpha)} \int_{0}^{t}(t-s)^{\alpha-1} f(s) d s, f \in S_{F, \omega, x}\right\}
$$

is a random operator.

Proof. From $\left(\mathscr{I}_{1}\right)$ and $\left(\mathscr{I}_{2}\right)$, we see that $S_{F, \omega, x} \neq \emptyset$, so $G(\omega, x) \neq \emptyset$. Now fix $x(\cdot) \in C([0, b], X)$ and consider $\omega \rightarrow G(\omega, x)$. We will show that $G(\cdot, x)$ is measurable. Let $x^{*} \in X^{*}$. Then

$$
\sigma\left(x^{*}, \Phi(\omega, t)\right)=\frac{1}{\Gamma(\alpha)} \int_{0}^{t}(t-s)^{\alpha-1} \sigma\left(x^{*}, F(\omega, s, x(s)) d s\right.
$$

where

$$
\Phi(\omega, t)=\frac{1}{\Gamma(\alpha)} \int_{0}^{t}(t-s)^{\alpha-1} F(s, \omega, x(s)) d s .
$$

By $\left(\mathscr{I}_{1}\right)$ and $\left(\mathscr{I}_{2}\right),(\omega, t) \rightarrow \sigma\left(x^{*}, F(\omega, t, x(t))\right.$ and is integrally bounded in $t$. This implies that $(\omega, t) \rightarrow \sigma\left(x^{*}, \Phi(\omega, t)\right)$ is a random variable. Let $t_{1}, t_{2} \in[0, b]$ with $t_{1} \leq t_{2}$. Then

$$
\begin{aligned}
\left\|\Phi\left(\omega, t_{2}\right)-\Phi\left(\omega, t_{1}\right)\right\|_{\mathscr{P}} \leq & \frac{\left\|\gamma_{*}(\omega, \cdot)\right\|_{\infty}}{\Gamma(\alpha)} \int_{0}^{t_{1}}\left(\left(t_{2}-s\right)^{\alpha-1}-\left(t_{1}-s\right)^{\alpha-1}\right) d s \\
& +\frac{\left\|\gamma_{*}(\omega, \cdot)\right\|_{\infty}}{\Gamma(\alpha)} \int_{t_{1}}^{t_{2}}\left(t_{2}-s\right)^{\alpha-1} d s \\
\leq & \frac{\left\|\gamma_{*}(\omega, \cdot)\right\|_{\infty}}{\Gamma(\alpha+1)} t_{2}^{\alpha}-\frac{\left\|\gamma_{*}(\omega, \cdot)\right\|_{\infty}}{\Gamma(\alpha+1)} t_{1}^{\alpha} \rightarrow 0 \quad \text { as }\left|t_{1}-t_{2}\right| \rightarrow 0 .
\end{aligned}
$$

From $\left(\mathscr{I}_{2}\right)$, we have

$$
\begin{aligned}
\left|\sigma\left(x^{*}, \Phi\left(\omega, t_{2}\right)\right)-\sigma\left(x^{*}, \Phi\left(\omega, t_{1}\right)\right)\right| \\
\left.\leq \frac{1}{\Gamma(\alpha)} \int_{t_{1}}^{t_{2}}\left(t_{2}-s\right)^{\alpha-1}\right) \mid \sigma\left(x^{*}, F(\omega, s, x(\omega, s)) \mid d s\right. \\
\quad+\frac{1}{\Gamma(\alpha)} \mid \int_{0}^{t_{1}}\left(\left(t_{1}-s\right)^{\alpha-1}-\left(t_{2}-s\right)^{\alpha-1}\right) \sigma\left(x^{*}, F(\omega, s, x(\omega, s)) d s \mid\right. \\
\leq \frac{\left\|\gamma_{*}(\omega, .)\right\|_{\infty}}{\Gamma(\alpha+1)}\left\|x^{*}\right\|\left(t_{2}-t_{1}\right)^{\alpha}+\left\|x^{*}\right\|\left\|\Phi\left(\omega, t_{2}\right)-\Phi\left(\omega, t_{1}\right)\right\|_{\mathscr{P} \rightarrow 0}
\end{aligned}
$$

as $\left|t_{1}-t_{2}\right| \rightarrow 0$. Therefore, one has $(\omega, t) \rightarrow \sigma\left(x^{*}, \Phi(\omega, t)\right)$ is a Carathéodory function and so it is $\mathscr{B}([0, b]) \otimes \Sigma$ - measurable. Since $\Phi(\cdot, \cdot) \in \mathscr{P}(X)$, we see from [31, Theorem III-37] that $(\omega, t) \rightarrow \Phi(\omega, t)$ is $\Sigma \times \Sigma_{b}$-measurable. It is clear that

$$
G_{1}(\omega)=\left\{h \in C([0, b], X): d\left(h(\cdot), S_{\Phi(\cdot, \omega)}\right)=0\right\}=G(\omega, \cdot)
$$


Observe that

$$
\begin{aligned}
d\left(h(\cdot), S_{\Phi(\cdot, \omega)}\right) & =\inf _{y \in S_{\Phi(\cdot, \omega)}} \int_{0}^{b}|h(t)-y(t)| d t \\
& =\int_{0}^{b} \inf _{y(t) \in S_{\Phi(\omega, t)}}|h(t)-y(t)| d t \\
& =\int_{0}^{b} d(h(t), \Phi(\omega, t)) d t .
\end{aligned}
$$

Clearly, $(\omega, t) \rightarrow d(h(t), \Phi(\omega, t))$ is a $\Sigma \times \Sigma_{b}$-measurable function and $z \rightarrow d(z, \Phi(\omega, t))$ is a continuous function. Hence, $(\omega, t, z) \rightarrow d(z, \Phi(\omega, t))$ is a Carathéodory function. Thus, $(\omega, t) \rightarrow d(h(t), \Phi(\omega, t))$ is measurable, so we have that

$$
(\omega, h(\cdot)) \rightarrow d(h(\cdot), \Phi(\cdot, \omega))
$$

is a Carthéodory function and is $\Sigma \otimes \mathscr{B}(C([0, b], X))$-measurable. We conclude that

$$
G r\left(G_{1}\right)=\{(\omega, h) \in \Sigma \times C([0, b], X): d(h(\cdot), \Phi(\cdot, \omega))=0\} \in \Sigma \otimes \mathscr{B}(C([0, b], X)) .
$$

Therefore, $\omega \rightarrow G(\omega, x)$ is measurable and so $G$ is a random variable.

We will make use of the following conditions in the sequel:

$\left(\mathscr{I}_{3}\right)$ The mapping $p(\cdot, \omega, x)$ is continuous for all $(\omega, x) \in \Omega \times \mathbb{R}$, and there exists $a \in[0,1)$ such that

$$
|p(\omega, t, u)-p(\omega, t, v)| \leq a|u-v| \quad \text { for } t \in J, u, v \in \mathbb{R}, \omega \in \Omega \text {. }
$$

$\left(\mathscr{I}_{4}\right)$ There exist constants $c_{1}$ and $c_{2}$ such that

$$
\|F(\omega, t, v)\|_{\mathscr{P}} \leq c_{1}|v|+c_{2} \text { for }(\omega, t, v) \in \Omega \times J \times \mathbb{R} .
$$

$\left(\mathscr{I}_{5}\right)$ For all $(\omega, t) \in \Omega \times J, F(\omega, t, \cdot)$ is u.s.c., and for all $x \in \mathbb{R}, F(\cdot, \cdot, x)$ is jointly measurable. Let $\mathscr{N}: \Omega \times C(J, \mathbb{R}) \rightarrow \mathscr{P}(C(J, \mathbb{R}))$ be the operator defined by

$$
\mathscr{N}(\omega, x(\cdot, \omega))=G(\omega, x(\cdot))+B(\omega, x(\cdot)), \quad x \in C(J, \mathbb{R}), \omega \in \Omega,
$$

where $G$ and $B$ are defined in (5.2) and (5.3) above. For fixed $\omega \in \Omega$, we set

$$
\mathscr{N}_{\omega}(x)=G_{\omega}(x)+B_{\omega}(x), \quad x \in C(J, \mathbb{R}),
$$

where

$$
G_{\omega}(x)=G(\omega, x) \text { and } B_{\omega}(x)=B(\omega, x), x \in \mathbb{R} .
$$


The proof of the following form of Gronwall's lemma for singular kernels can be found in [32, Lemma 7.1.1].

Lemma 5.3. Let $v:[0, b] \rightarrow[0, \infty)$ be a real function and $w:[0, b] \rightarrow[0, \infty)$ be a nonnegative locally integrable function. Assume that there are constants $a>0$ and $0<\gamma<1$ such that

$$
v(t) \leq w(t)+a \int_{0}^{t} \frac{v(s)}{(t-s)^{\gamma}} d s
$$

Then there exists a constant $K=K(\gamma)$ such that

$$
v(t) \leq w(t)+K(\gamma) a \int_{0}^{t} \frac{w(s)}{(t-s)^{\gamma}} d s
$$

for every $t \in[0, b]$.

Before presenting the main result in this section, we first prove some lemmas that will be used in the sequel.

Lemma 5.4. Under conditions $\left(\mathscr{I}_{3}\right)-\left(\mathscr{I}_{4}\right), B_{\omega}$ is a contraction operator and $G_{\omega}$ is completely continuous, u.s.c., and $G_{\omega}(\cdot, \cdot) \in \mathscr{P}_{c p, c v}(C(J, \mathbb{R}))$.

Proof. We first show that $B_{\omega}$ is a contraction operator and $G_{\omega}$ is completely continuous. The proof will be given in several steps.

Step 1. $B_{\omega}(\cdot)$ is contractive. Let $x, \tilde{x} \in C([0, b], \mathbb{R})$. Then

$$
\left|B_{\omega}(x(t))-B_{\omega}(\widetilde{x}(t))\right|=|p(\omega, t, x(\omega, t))-p(\omega, t, \widetilde{x}(\omega, t))| \leq a|x(\omega, t)-\widetilde{x}(\omega, t)| .
$$

Hence,

$$
\left\|B_{\omega}(x)-B_{\omega}(\widetilde{x})\right\|_{\infty} \leq a\|x(\omega, \cdot)-\widetilde{x}(\omega, \cdot)\|_{\infty} .
$$

Step 2. $G_{\omega}$ maps bounded sets in $C(J, \mathbb{R})$ into equicontinuous sets in $C(J, \mathbb{R})$. Let $r>0$ and $\bar{B}_{r}:=\left\{x \in C(J, \mathbb{R}):\|x\|_{\infty} \leq r\right\}$ be a bounded set in $C(J, \mathbb{R}), 0<\tau_{1}<\tau_{2} \leq b$, and $x \in B_{r}$. For each $h \in G_{\omega}(x)$, we have

$$
\begin{aligned}
\left|h\left(\tau_{2}\right)-h\left(\tau_{1}\right)\right| & \leq \frac{\left(c_{1} r+c_{2}\right)}{\Gamma(\alpha)} \int_{0}^{\tau_{1}}\left(\left(\tau_{2}-s\right)^{\alpha-1}-\left(\tau_{1}-s\right)^{\alpha-1}\right) d s+\frac{\left(c_{1} r+c_{2}\right)}{\Gamma(\alpha)} \int_{\tau_{1}}^{\tau_{2}}\left(\tau_{2}-s\right)^{\alpha-1} d s \\
& \leq \frac{\left(c_{1} r+c_{2}\right)}{\Gamma(\alpha+1)}\left[\tau_{2}^{\alpha}-\tau_{1}^{\alpha}\right] .
\end{aligned}
$$

Now the right hand side tends to zero as $\tau_{2}-\tau_{1} \rightarrow 0$. As a consequence of Step 1 together with the Arzelà-Ascoli theorem, we conclude that $N$ maps $\bar{B}_{r}$ into a precompact set in $C([0, b], \mathbb{R})$. Thus, $G_{\omega}$ is completely continuous. 
Step 3. Since for each $x \in C(J, \mathbb{R})$, the nonlinearity $F$ takes convex values, the selection set $S_{F, \omega, x}$ is convex, and by $\left(\mathscr{I}_{4}\right), G_{\omega}$ has convex values. Using $\left(\mathscr{I}_{4}\right)$, we can prove that $G_{\omega}$ maps bounded sets into equicontinuous sets, and as in Step 2, we can easily prove that $G_{\omega}(\cdot, \cdot) \in$ $\mathscr{P}_{c p, c v}(C(J, \mathbb{R})$.

Lemma 5.5. Assume that $\left(\mathscr{I}_{3}\right)-\left(\mathscr{I}_{4}\right)$ hold. Then every solution $x \in C(J, \mathbb{R})$ of the inclusion

$$
x(\omega, t) \in \lambda G(\omega, x(\omega, t))+\lambda B\left(\omega, \frac{x(\omega, t)}{\lambda}\right), \text { for some } 0<\lambda<1,
$$

is bounded.

Proof. Let $x(\omega, t) \in C(J, \mathbb{R})$ be a solution of (5.6). Then there exists $f \in S_{F, \omega, x}$ such that

$$
x(\omega, t)=\lambda x_{0}(\omega, t)+\frac{\lambda}{\Gamma(\alpha)} \int_{0}^{t}(t-s)^{\alpha-1} f(s) d s+\lambda p(\omega, t, x(\omega, t)) .
$$

Thus,

$$
\begin{aligned}
|x(\omega, t)| & \leq\left\|x_{0}(\omega, \cdot)\right\|_{\infty}+\frac{1}{\Gamma(\alpha)} \int_{0}^{t}(t-s)^{\alpha-1}|f(s)| d s+|p(\omega, t, x(\omega, t))| \\
& \leq\left\|x_{0}(\omega, \cdot)\right\|_{\infty}+\frac{1}{\Gamma(\alpha)} \int_{0}^{t}(t-s)^{\alpha-1}\left(c_{1}|x(\omega, s)|+c_{2}\right) d s+|p(\omega, t, 0)|+a|x(\omega, t)|
\end{aligned}
$$

Hence,

$$
\begin{aligned}
|x(\omega, t)| \leq & \frac{1}{1-a}\left(\left\|x_{0}(\omega, \cdot)\right\|_{\infty}\right. \\
& \left.+\|p(\omega, \cdot, 0)\|_{\infty}+\frac{1}{\Gamma(\alpha)} \int_{0}^{t}(t-s)^{\alpha-1} c_{1}|x(\omega, s)| d s+\frac{c_{2}}{\Gamma(\alpha+1)}\right) .
\end{aligned}
$$

By Lemma 5.3, there exists $K(\alpha)>0$ such that

$$
|x(\omega, t)| \leq c_{*}+K_{*}(\alpha) \int_{0}^{t}(t-s)^{\alpha-1} d s
$$

where

$$
c_{*}=\frac{1}{1-a}\left(\left\|x_{0}(\omega)\right\|_{\infty}+\|p(\omega, \cdot, 0)\|_{\infty}+\frac{c_{2}}{\Gamma(\alpha+1)}\right), \quad K_{*}(\alpha)=\frac{c_{1} K(\alpha)}{(1-a) \Gamma(\alpha)} .
$$

Consequently, $\|x(\omega, \cdot)\|_{\infty} \leq c_{*}+K_{*}(\alpha):=K_{*}$.

We are now in the position to prove our main existence result for the inclusion (5.1).

Theorem 5.1. If $\left(\mathscr{I}_{3}\right)-\left(\mathscr{I}_{5}\right)$ hold, then $(5.1)$ has a random solution in $C(J, \mathbb{R})$. 
Proof. Fixing $\omega \in \Omega$, let $\mathscr{N}_{\omega}: C(J, \mathbb{R}) \rightarrow \mathscr{P}_{c p, c v}(C(J, \mathbb{R}))$ be the multivalued operator defined by

$$
\mathscr{N}_{\omega}(x)=G_{\omega}(x)+B_{\omega}(x), \quad x \in C(J, \mathbb{R}),
$$

where $G_{\omega}$ and $B_{\omega}$ are defined in (5.4) and (5.5) above. We now show that all the conditions of Theorem 4.5 are satisfied.

First observe from Lemma 5.4 that operator $B_{\omega}$ is contractive and $G_{\omega}: C(J, \mathbb{R}) \rightarrow \mathscr{P}_{c p, c v}(C(J, \mathbb{R}))$ is completely continuous.

Step 1. $\mathscr{N}$ is random variable. From Lemmas 5.1 and 5.2, $B$ and $G$ are random variables, so $\mathscr{N}$ is random variable.

Step 2. $G_{\omega}$ is u.s.c. Since $G_{\omega}$ is completely continuous, we only need to prove that $G_{\omega}$ is closed. Let $h_{n} \longrightarrow h_{*}$ with $h_{n} \in G_{\omega}\left(x_{n}\right)$, and $x_{n} \longrightarrow x$. We need to show that $h_{*} \in G_{\omega}(x)$. Now $h_{n} \in G_{\omega}\left(x_{n}\right)$ means that there exists $f_{n} \in S_{F, \omega, x_{n}}$ such that, for a.e. $t \in J$,

$$
h_{n}(t)=\frac{1}{\Gamma(\alpha)} \int_{0}^{t}(t-s)^{\alpha-1} f_{n}(s) d s .
$$

Since $\left\{f_{n}(t): n \in \mathbb{N}\right\} \subseteq F\left(\omega, t, x_{n}(t)\right)$, condition $\left(\mathscr{I}_{4}\right)$ implies that $\left\{f_{n}\right\}$ is integrally bounded and there exists $K>0$ such that

$$
\int_{0}^{b}\left|f_{n}(s)\right| d s \leq K, \quad \text { for each } n \in \mathbb{N} .
$$

Then by the Dunford-Pettis Theorem, $\left\{f_{n}(t): n \in \mathbb{N}\right\}$ is weakly compact in $L^{1}(J, \mathbb{R})$. So by passing to a subsequence if necessary, we may assume that $\left\{f_{n}\right\}_{n \in \mathbb{N}} \in L^{1}(J, \mathbb{R})$ and $\left\{f_{n}\right\} \rightarrow$ $f_{*} \in L^{1}(J, \mathbb{R})$. Mazur's Lemma implies the existence of a double sequence $\left\{\alpha_{m, k}\right\}_{k, m \in \mathbb{N}}$ such that for every $m \in \mathbb{N}$ there exists $k_{0}(m) \in \mathbb{N}$ such that $\alpha_{m, k}=0$ for all $k \geq k_{0}(n), \sum_{k=m}^{\infty} \alpha_{m, k}=1$ for all $m \in \mathbb{N}$, and the sequence of convex combinations $g_{m}(\cdot)=\sum_{k=n}^{\infty} \alpha_{m, k} f_{k}(\cdot)$ converges strongly to $f_{n}$ in $L^{1}$. Since $F(\omega, t, \cdot)$ is u.s.c., given $\varepsilon>0$ we can find $m \in \mathbb{N}$ such that, for $n \geq m$, we have

$$
F\left(\omega, t, x_{n}(\omega, t)\right) \subset F(\omega, t, x(\omega, t))+\varepsilon \bar{B}(0,1) .
$$

It follows that

$$
\overline{c o} \bigcup_{n \geq m} F\left(\omega, t, x_{n}(\omega, t)\right) \subseteq \overline{c o} F(\omega, t, x(\omega, t))+\varepsilon \bar{B}(0,1) .
$$

Hence, $g_{m}(t) \in \overline{c o} F(\omega, t, x(\omega, t))+\varepsilon \bar{B}(0,1)$. Taking the limit as $m \rightarrow \infty$, we obtain

$$
f_{*}(t) \in \overline{c o} F(\omega, t, x(\omega, t))+\varepsilon \bar{B}(0,1) .
$$


Letting $\varepsilon \rightarrow 0$, we find

$$
f_{*}(t) \in \overline{c o} F(\omega, t, x(\omega, t)) \text { implies } f_{*}(t) \in F(\omega, t, x(\omega, t)) .
$$

Hence, $f_{*} \in S_{F, \omega, x *}$, and for each $t \in J$,

$$
h_{*}(t)=\frac{1}{\Gamma(\alpha)} \int_{0}^{t}(t-s)^{\alpha-1} f_{*}(s) d s .
$$

As a consequence, $h_{*} \in G_{\omega}(x)$, and this implies that $G_{\omega}$ is closed.

Step 3. It remains to show that the set

$$
\mathscr{M}(\omega)=\left\{x(\omega, \cdot) \in C([0, b], \mathbb{R}): x(\omega, \cdot) \in \lambda G_{\omega}(x)+\lambda B_{\omega}\left(\frac{x}{\lambda}\right), \lambda \in(0,1)\right\}
$$

is bounded. By Lemma 5.5, $\|x(\omega, .)\|_{\infty} \leq K_{*}$. So $\mathscr{M}(\omega)$ is bounded. As a consequence of Theorem 4.5, we have that $\mathscr{N}$ has a random fixed point $t \rightarrow x(\omega, t)$ that in turn is a solution to (5.1).

Remark 5.1. We can replace $\left(\mathscr{I}_{4}\right)$ by the condition

$\left(\overline{\mathscr{I}}_{4}\right)$. There exist measurable functions $\gamma_{1}, \gamma_{2}: \Omega \rightarrow C\left(J, \mathbb{R}_{+}\right)$such that

$$
\|F(\omega, t, x)\|_{\mathscr{P}} \leq \gamma_{1}(\omega, t)|x|+\gamma_{2}(\omega, t), \quad \text { for all } t \in J, \omega \in \Omega
$$

We conclude this section by pointing out that some existence results for random fractional differential equations on compact intervals can be found in the literature; see, for example, $[29,30]$ and the references therein. Our work here gives multivalued version for such results.

\section{Random fixed points for a Cartesian product of operators}

In this section, we prove the existence of solutions to the system

$$
\left\{\begin{array}{l}
x=F(\omega, x, y), \\
y=G(\omega, x, y) .
\end{array}\right.
$$

Let $\left(X,\|\cdot\|_{X}\right.$ and $\left(Y,\|\cdot\|_{Y}\right)$ be two Banach spaces and let $F: \Omega \times X \times Y \rightarrow X$ and $G: \Omega \times X \times$ $Y \rightarrow Y$.

We begin with a result for a special case of system (6.1).

Theorem 6.1. Assume that: 
$\left(\mathscr{S}_{1}\right)$ For every $x \in X$, the function $y \rightarrow F(x, y)$ is a continuous operator;

$\left(\mathscr{S}_{2}\right)$ There exists $k>1$ such that

$$
\left\|F\left(x_{1}, y\right)-F\left(x_{2}, y\right)\right\|_{X} \geq k\left\|x_{1}-x_{2}\right\| \quad \text { for every } x_{1}, x_{2} \in X, y \in Y
$$

and for each $y \in Y$,

$$
X \subset F(X, y)
$$

$\left(\mathscr{S}_{3}\right) G(\cdot, \cdot)$ is a continuous compact operator.

Then the system

$$
\left\{\begin{array}{l}
x=F(x, y), \\
y=G(x, y),
\end{array}\right.
$$

admits a solution

Proof. Fix $y \in Y$ and define $T_{y}: X \rightarrow X$ by $T_{y}(x)=F(x, y)$. In view of $\left(\mathscr{T}_{2}\right)$, we can apply Theorem 3.1 to obtain the existence of a unique $x(y) \in X$ such that $x(y)=T_{y}(x(y))$. Therefore, for every $y \in Y$, there exists a unique $x(y) \in X$ such that $x(y)=F(x(y), y)$. Define $\bar{T}: Y \rightarrow Y$ by $\bar{T}(y)=G(x(y), y)$ for each $y \in Y$. We shall show that $\bar{T}$ is a continuous compact operator. Let $\left\{y_{n}\right\}$ be a sequence converging to $y$ in $Y$. Then,

$$
\begin{aligned}
\left\|x\left(y_{n}\right)-x(y)\right\|_{X} & =\left\|F\left(x\left(y_{n}\right), y_{n}\right)-F(x(y), y)\right\|_{X} \\
& \geq\left\|F\left(x\left(y_{n}\right), y_{n}\right)-F\left(x(y), y_{n}\right)\right\|_{X}-\left\|F\left(x(y), y_{n}\right)-F(x(y), y)\right\|_{X} \\
& \left.\geq k \| x\left(y_{n}\right)-x(y)\right)\left\|_{X}-\right\| F\left(x(y), y_{n}\right)-F(x(y), y) \|_{X} .
\end{aligned}
$$

It follows that

$$
\left\|x\left(y_{n}\right)-x(y)\right\|_{X} \leq \frac{1}{k-1}\left\|F\left(x(y), y_{n}\right)-F(x(y), y)\right\|_{X} \rightarrow 0 \text { as } n \rightarrow \infty .
$$

Thus, $x(\cdot)$ is continuous, and so by $\left(\mathscr{S}_{3}\right), \bar{T}$ is continuous and compact. Therefore, by Schauder's fixed point theorem, there exists $y \in Y$ such that

$$
y=G(x(y), y)
$$

We then have that $(x(y), y)$ is a solution of problem (6.2), and this proves the theorem.

Our next two results are for system (6.1). 
Theorem 6.2. Let $(\Omega, \Sigma)$ be a measurable space. Let $\left(X,\|\cdot\|_{X}\right)$ and $\left(Y,\|\cdot\|_{Y}\right)$ be two separable Banach spaces. Let $F: \Omega \times X \times Y \rightarrow X$ and $G: \Omega \times X \times Y \rightarrow Y$ be random operators. Assume that:

$\left(\mathscr{S}_{4}\right)$ For every $\omega \in \Omega$, the function $(x, y) \rightarrow F(\omega, x, y)$ is a continuous operator;

$\left(\mathscr{S}_{5}\right)$ There exists $k(\omega)>1$ such that

$\left\|F\left(\omega, x_{1}, y\right)-F\left(\omega, x_{2}, y\right)\right\|_{X} \geq k(\omega)\left\|x_{1}-x_{2}\right\|$ for every $x_{1}, x_{2} \in X, y \in Y, \omega \in \Omega$, and for each $y \in Y$ and $\omega \in \Omega$, we have

$$
X \subset F(\omega, X, y)
$$

$\left(\mathscr{S}_{6}\right)$ For every $\omega \in \Omega, G(\omega, \cdot, \cdot)$ is a continuous compact operator.

Then system (6.1) has at least one random solution.

Proof. Let $y \in Y$ and $\omega \in \Omega$. Consider $T_{\omega, y}: X \rightarrow X$ defined by $T_{\omega, y}(x)=F(\omega, x, y)$. By Theorem 3.1, there exists a unique $x(y)(\omega) \in X$ such that

$$
x(y)(\omega)=F(\omega, x(y)(\omega), y) .
$$

Define $\mathscr{T}_{y}: X \rightarrow \mathscr{P}_{c l}(X)$ by

$$
\mathscr{T}_{y}(\omega)=\{x \in X: x=F(\omega, x, y)\}
$$

Since $F(\omega, \cdot, y)$ is continuous, the function $\varphi: X \rightarrow \mathbb{R}$ be defined by $\phi(x)=d(x, x-F(\omega, x, y))$ is measurable. Hence, $\mathscr{T}_{y}$ has a measurable selection.

Now, let $T: Y \rightarrow Y$ be defined by $T(y)=G(\omega, x(y)(\omega), y)$. It is clear that $T$ is a continuous operator. Thus, from Schauder's fixed point theorem, there exist $y(\omega) \in Y$ such that

$$
y(\omega)=G(\omega, x(y)(\omega), y(\omega)), \omega \in \Omega .
$$

Using the fact that $G(\cdot, x, y)$ is measurable and $G(\omega, \cdot, \cdot)$ is continuous, we see that $y: \Omega \rightarrow Y$ is a measurable function. Hence, $(x(y)(\omega), y(\omega))$ is a solution of problem (6.1).

We can also prove the following result.

Theorem 6.3. Let $(\Omega, \Sigma)$ be a measurable space. Let $\left(X,\|\cdot\|_{X}\right)$ and $\left(Y,\|\cdot\|_{Y}\right)$ be two separable Banach spaces. Let $F: \Omega \times X \times Y \rightarrow X$ and $G: \Omega \times X \times Y \rightarrow Y$ be two random operators. Assume that: 
$\left(\overline{\mathscr{S}}_{1}\right)$ For every $\omega \in \Omega$, the function $(x, y) \rightarrow F(\omega, x, y)$ is a continuous operator;

$\left(\overline{\mathscr{S}}_{2}\right)$ There exists $k(\omega) \in[0,1)$ such that

$$
\left\|F\left(x_{1}, y\right)-F\left(x_{2}, y\right)\right\|_{X} \leq k(\omega)\left\|x_{1}-x_{2}\right\| \text { for every } x_{1}, x_{2} \in X, y \in Y, \omega \in \Omega
$$

$\left(\overline{\mathscr{S}}_{3}\right)$ For every $\omega \in \Omega, G(\omega, \cdot, \cdot)$ is a continuous compact operator.

Then system (6.1) has at least one random solution.

\section{Random fixed points in vector metric spaces}

The classical Banach contraction principle was extended to contractive maps on spaces endowed with a vector-valued metric by Perov in 1964 [33] and Perov and Kibenko [34]. Schauder's fixed point theorem was extended to generalized Banach spaces by Viorel [35]. Very recently, in vector-valued Banach spaces, Krasnosel'skii type fixed point theorems for single and multivalued operators were studied by Petre and Petrussel [36], and Petre [37]. In [38], the authors obtained a random version of the Perov and Schauder fixed point theorems. The goal of this section is to give a multivalued random version of the Perov and Schauder fixed point theorems in separable vector metric spaces.

Definition 7.1. Let $X$ be a nonempty set. By a vector-valued metric on $X$ we mean a map $d: X \times X \rightarrow \mathbb{R}^{n}$ satisfying:

(i) $d(u, v) \geq 0$ for all $u, v \in X$, and if $d(u, v)=0$, then $u=v$;

(ii) $d(u, v)=d(v, u)$ for all $u, v \in X$;

(iii) $d(u, v) \leq d(u, w)+d(w, v)$ for all $u, v, w \in X$.

We call the pair $(X, d)$ a generalized metric space with $d(x, y):=\left(\begin{array}{c}d_{1}(x, y) \\ \vdots \\ d_{n}(x, y)\end{array}\right)$. Notice that $d$ is a generalized metric space on $X$ if and only if $d_{i}, i=1, \ldots, n$, are metrics on $X$.

For $r=\left(r_{1}, \ldots, r_{n}\right) \in \mathbb{R}_{+}^{n}$, we let

$$
B\left(x_{0}, r\right)=\left\{x \in X: d\left(x_{0}, x\right)<r\right\}
$$


denote the open ball centered at $x_{0}$ with radius $r$, and

$$
\overline{B\left(x_{0}, r\right)}=\left\{x \in X: d\left(x_{0}, x\right) \leq r\right\}
$$

be the closed ball centered at $x_{0}$ with radius $r$. We mention that for a generalized metric space, the notions of open set, closed set, convergence, Cauchy sequence, and completeness are similar to those in usual metric spaces.

A classic result in matrix analysis is contained in the following theorem; see $[39,40]$ and the references therein.

Theorem 7.1. Let $M \in \mathscr{M}_{n \times n}\left(\mathbb{R}_{+}\right)$. The following assertions are equivalent:

(i) $M \rightarrow 0$ ( $M$ is convergent to 0$)$;

(ii) $M^{k} \rightarrow 0$ as $k \rightarrow \infty$;

(iii) The matrix $(I-M)$ is nonsingular and

$$
(I-M)^{-1}=I+M+M^{2}+\cdots+M^{k}+\cdots
$$

(iv) The matrix $(I-M)$ is nonsingular and $(I-M)^{-1}$ has nonnegative elements.

Let $\left(X, d_{*}\right)$ be a metric space; we will denote by $H_{d_{*}}: \mathscr{P}(X) \times \mathscr{P}(X) \longrightarrow \mathbb{R}_{+} \cup\{\infty\}$ the usual Hausdorff-Pompeiu metric on $\mathscr{P}(X)$ defined by

$$
H_{d_{*}}(A, B)=\max \left\{\sup _{a \in A} d_{*}(a, B), \sup _{b \in B} d_{*}(A, b)\right\}
$$

where $d_{*}(A, b)=\inf _{a \in A} d_{*}(a, b)$ and $d_{*}(a, B)=\inf _{b \in B} d_{*}(a, b)$. Then $\left(\mathscr{P}_{b, c l}(X), H_{d_{*}}\right)$ is a metric space and $\left(\mathscr{P}_{c l}(X), H_{d_{*}}\right)$ is a generalized metric space. In particular, $H_{d_{*}}$ satisfies the triangle inequality.

We also consider the generalized Hausdorff pseudo-metric

$$
H_{d}: \mathscr{P}(X) \times \mathscr{P}(X) \longrightarrow \mathbb{R}_{+}^{n} \cup\{\infty\}
$$

defined by

$$
H_{d}(A, B):=\left(\begin{array}{c}
H_{d_{1}}(A, B) \\
\vdots \\
H_{d_{n}}(A, B)
\end{array}\right)
$$

We also need the following notions. 
Definition 7.2. If $\mathscr{U}$ and $\mathscr{V}$ are two covers of a topological space $X$, we will write $\mathscr{U}<\mathscr{V}$ to mean that each $U \in \mathscr{U}$ is contained in some $V \in \mathscr{V}$.

Definition 7.3. Let $X$ be a topological space and $\mathscr{U}$ be a collection of subsets of $X$. We say that $\mathscr{U}$ is locally finite if for every $x \in X$ there exists a neighborhood $V_{x}$ of $x$ such that $\{U \in \mathscr{U}$ : $\left.V_{x} \cap U \neq \emptyset\right\}$ is a finite set.

Definition 7.4. A topological space $X$ is paracompact if every open covering admits a locally finite refinement.

Definition 7.5. Let $(X, d)$ be a generalized metric space. A multivalued operator $N: X \rightarrow$ $\mathscr{P}_{c l}(X)$ is said to be contractive if there exists a matrix $M \in \mathscr{M}_{n \times n}\left(\mathbb{R}_{+}\right)$such that $M^{k} \rightarrow 0$ as $k \rightarrow \infty$ and $H_{d}(N(u), N(v)) \leq M d(u, v)$, for all $u, v \in X$

Definition 7.6. Let $(X, d)$ be a generalized metric space. A multivalued operator $N: X \rightarrow$ $\mathscr{P}_{c l}(X)$ is said to be contractive if there exists a matrix $M \in \mathscr{M}_{n \times n}\left(\mathbb{R}_{+}\right)$such that $M^{k} \rightarrow 0$ as $k \rightarrow \infty$ and $H_{d}(N(u), N(v)) \leq M d(u, v)$, for all $u, v \in X$.

Lemma 7.1. ([36]) Let $(X, d)$ be a generalized metric space, $A, B \in \mathscr{P}(X)$, and $b>1$. Then for every $u \in A$ there exists $v \in B$ such that $d(u, v) \leq b H_{d}(A, B)$.

Lemma 7.2. ([36]) Let $M \in \mathscr{M}_{n \times n}\left(\mathbb{R}_{+}\right)$be a matrix that converges to zero. Then there exists $a>1$ such that for any $b \in(1, a)$ we have that $b M \rightarrow 0$.

First we prove a random version of Nadler's fixed point theorem in a vector metric space.

Theorem 7.2. Let $(X, d)$ be a generalized complete separable metric space, and let $F: \Omega \times X \rightarrow$ $\mathscr{P}_{c l}(X)$ be a random multivalued map. Assume that, for every $\omega \in \Omega$, there exists $M(\omega) \in$ $\mathscr{M}_{n \times n}\left(\mathbb{R}_{+}\right)$such that $M(\omega) \rightarrow 0$ and

$$
H_{d}(F(\omega, x), F(\omega, y)) \leq M(\omega) d(x, y), \text { for } x, y \in X, \omega \in \Omega
$$

Then there exists a random function $x: \Omega \rightarrow X$ such that

$$
x(\omega) \in F(\omega, x(\omega)), \quad \omega \in \Omega
$$


Proof. Letting $y: \Omega \rightarrow X$ be any arbitrary measurable function, we set $x_{0}=y$ and $x_{1}(\omega) \in$ $F\left(\omega, x_{0}(\omega)\right)$. Let $b \in(1, a)$. By Lemma 7.1, there exists $x_{2}(\omega) \in F\left(\omega, x_{1}(\omega)\right)$ such that

$$
\begin{aligned}
d\left(x_{1}, x_{2}\right) & \leq b H_{d}\left(F\left(\omega, x_{1}(\omega)\right), F\left(\omega, x_{0}(\omega)\right)\right. \\
& \leq b M(\omega) d\left(x_{1}(\omega), x_{0}(\omega)\right) .
\end{aligned}
$$

Continuing this procedure we can construct a sequence $\left(x_{n}\right)_{n \in \mathbb{N}}$ in $X$ such that

$$
d\left(x_{n}(\omega), x_{n+1}(\omega)\right) \leq(b M(\omega))^{n}(\omega) d\left(x_{0}(\omega), x_{1}(\omega)\right), n \in \mathbb{N} .
$$

Let $p \in \mathbb{N}$. Since $d$ is a metric, we have

$$
d\left(x_{n}(\omega), x_{n+p}(\omega)\right) \leq d\left(x_{n}(\omega), x_{n+1}(\omega)\right)+\cdots+d\left(x_{n+p-1}(\omega), x_{n+p}(\omega)\right) .
$$

Hence, for all $n, p \in \mathbb{N}$, the following estimation holds:

$$
d\left(x_{n}(\omega), x_{n+p}(\omega)\right) \leq(b M(\omega))^{n}\left(I+b M+(b M(\omega))^{2}+\cdots+(b M(\omega))^{p-1}\right) d\left(x_{0}(\omega), x_{1}(\omega)\right) .
$$

Therefore, in view of Theorem 7.1(ii), one has $d\left(x_{n}(\omega), x_{n+p}(\omega)\right) \rightarrow 0$ as $n \rightarrow \infty$. So $\left(x_{n}(\omega)\right)_{n \in \mathbb{N}}$ is a Cauchy sequence in the complete generalized metric space $X$. Thus, there exists $x(\omega) \in X$ such that $d\left(x_{n}(\omega), x(\omega)\right) \rightarrow 0$ as $n \rightarrow \infty$. From (7.1), we obtain

$$
\begin{aligned}
d(x(\omega), F(\omega, x(\omega))) & \leq d\left(x(\omega), x_{n}(\omega)\right)+H_{d}\left(F\left(\omega, x_{n+1}(\omega)\right), F(\omega, x(\omega))\right) \\
& \leq d\left(x(\omega), x_{n}(\omega)\right)+M(\omega) d\left(x_{n+1}(\omega), x(\omega)\right) \rightarrow 0 \text { as } n \rightarrow \infty .
\end{aligned}
$$

This implies that $x(\omega) \in F(\omega, x(\omega))$, for every $\omega \in \Omega$. Clearly, $x_{0}$ is a random variable. So, for every $n \in \mathbb{N}, x_{n}: \Omega \rightarrow X$ is a random variable. Since $x$ is the limit of the sequence $\left(x_{n}\right)$, we find that $x: \Omega \rightarrow X$ is a random variable. This completes the proof.

The following continuous approximation theorem is a key result in this section.

Theorem 7.3. Let $(X,|\cdot|)$ be a generalized normed space. Let $(Y,\|\cdot\|)$ be a generalized Banach space and let $F: X \rightarrow \mathscr{P}_{c v}(Y)$ be an u.s.c. multivalued map. Then, for every $\varepsilon \in \mathbb{R}_{+}^{n}$, there exists a locally Lipschitzian function $f_{\varepsilon}: X \rightarrow Y$ such that $f_{\varepsilon}(X) \subseteq \operatorname{coF}(X)$ and $\mathscr{G} r\left(f_{\varepsilon}\right) \subseteq$ $\mathscr{G} r(F)+B(F(x), \varepsilon)$, where $B(F(x), \varepsilon)=\{z \in X: d(z, F(x))<\varepsilon\}$.

Proof. Fixing $\varepsilon=\left(\varepsilon_{1}, \ldots, \varepsilon_{n}\right)>0$, we see, for every $x \in X$, that there exists $B(x, \delta(x)) \subset X$ such that

$$
F(y) \subseteq F(B(x, \delta(x)) \subset F(x)+B(0, \varepsilon) \text { for each } y \in B(x, \delta(x)),
$$


where $\delta(x)=\left(\delta_{1}(x), \ldots, \delta_{n}(x)\right)>0$. We take $0<\delta(x) \leq \frac{\varepsilon}{2}$. The family $\mathscr{B}=\{B(x, \delta(x))\}_{x \in X}$ is an open cover of $X$. Clearly, $X$ is a paracompact space. Let $\left\{U_{i}\right\}_{i \in I_{*}}$ be a finite local refinement of $\mathscr{B}$ and let $\left\{f_{i}\right\}_{i \in I_{*}}$ be a locally Lipschitzean partition of unity subordinate to it. For each $i \in I_{*}$, choose $x_{i} \in U_{i}$ and define $f_{\varepsilon}$ by

$$
f_{\varepsilon}(x)=\sum_{i \in I_{*}} f_{i}(x) z_{i} \text { for each } x \in X,
$$

where $z_{i} \in F\left(x_{i}\right)$. It is clear that $f_{\varepsilon}$ is well defined, locally Lipschitzean, and $f_{\varepsilon}(X) \subseteq \operatorname{co}(F(X))$. Now we show that $f_{\varepsilon}$ is an approximation of $F$. Let $x \in X$ and $I_{*}(x)$ be the subset of all $i \in I_{*}$ such that $f_{i}(x) \neq 0$. Then, $x \in \bigcap_{i \in I_{*}(x)} B\left(x_{i}, \delta_{i}(x)\right)$. Letting $i, j \in I_{*}(x)$, one has

$$
d\left(x_{i}, x_{j}\right) \leq d\left(x_{i}, x\right)+d\left(x, x_{j}\right) \leq \delta_{i}+\delta_{j}<\varepsilon .
$$

Let $k \in I_{*}(x)$ be such that $\delta_{k}=\max _{i \in I_{*}} \delta_{i}$. Then, for every $i \in I_{*}(x)$, we have

$$
F\left(x_{i}\right) \subset F\left(B\left(x_{i}, \delta_{i}\right)\right) \subset F\left(B\left(x_{k}, 2 \delta_{k}\right)\right) \subset F\left(x_{k}\right)+B(0, \varepsilon) \text {, for all } i \in I_{*}(x) \text {. }
$$

Since $F(x)+B(0, \varepsilon)$ is convex, one obtains that $f_{\varepsilon}(x) \in F(x)+B(0, \varepsilon)$.

Next we state a version of Schauder's fixed point theorem in generalized Banach spaces [35].

Theorem 7.4. Let $E$ be a generalized Banach space, $C \subset E$ be a nonempty closed convex subset of $E$, and $N: C \rightarrow C$ be a continuous operator with a relatively compact range. Then $N$ possesses at least one fixed point in $C$.

The following result is a consequence of the Kuratowski-Ryll-Nardzewski selection theorem.

Theorem 7.5. ([38]) Let $(\Omega, \Sigma)$ be a measurable space, $Y$ be a separable generalized metric space, and $F: \Omega \rightarrow \mathscr{P}_{c l}(Y)$ be a measurable multivalued mapping. Then $F$ has a measurable selection.

By the same method used in [7], we can prove the following proposition.

Proposition 7.6. Let $(X, d)$ be a separable complete vector metric space, $f: \Omega \times X \rightarrow X$ be a Carathéodory mapping, $F: \Omega \rightarrow \mathscr{P}_{c l, b}(X)$ be a measurable multivalued mapping, and $U$ be an open subset of $X$. Then the mapping $G: \Omega \rightarrow \mathscr{P}(X)$ defined by $G(\omega)=\{\omega \in F(\omega): f(\omega, x) \in$ $U\}, \omega \in \Omega$, is measurable.

We also have the following proposition. 
Proposition 7.7. Let $F, G: \Omega \rightarrow \mathscr{P}_{c l, b}(X)$ be measurable mappings, $u: \Omega \rightarrow X$ be a measurable selector of $F$, and $r: \Omega \rightarrow \mathbb{R}_{+}^{n}$ be a measurable function. Then there exists a measurable selection $v: \Omega \rightarrow X$ of $G$ such that $d(u(\omega), v(\omega)) \leq H_{d}(F(\omega), G(\omega))+r(\omega), \omega \in \Omega$.

Proof. Since $F$ and $G$ are measurable, there exist countable families $\left(f_{m}\right)_{m \in \mathbb{N}}$ and $\left(g_{m}\right)_{m \in \mathbb{N}}$ of measurable selections of $F$ and $G$, respectively, such that $F(\omega)=\overline{\left\{f_{m}(\omega): m \in \mathbb{N}\right\}}, \omega \in \Omega$, and $G(\omega)=\overline{\left\{g_{m}(\omega): m \in \mathbb{N}\right\}}, \omega \in \Omega$. Hence, the real vector multifunction $H_{*}: \Omega \rightarrow \mathbb{R}_{+}^{n}$ defined by $H_{*}(\omega)=H_{d}(F(\omega), G(\omega)), \omega \in \Omega$, is measurable. Let $h: \Omega \times X \rightarrow \mathbb{R}$ and $L: \Omega \rightarrow \mathscr{P}(X)$ be defined by $h(\omega, x)=d(u(\omega), x)-H_{*}(\omega)-r(\omega)$ and $L(\omega)=\{\omega \in G(\omega): f(\omega, x)<0\}$. By Proposition 7.6, $L$ is measurable. Thus, $\bar{L}: \Omega \rightarrow \mathscr{P}_{c l, b}(X)$ defined by $\bar{L}(\omega)=\overline{L(\omega)}, \quad \omega \in \Omega$, is measurable, and by Theorem 7.5 has measurable selection $v: \Omega \rightarrow X$ such that $v(\omega) \in L(\omega)$, for any $\omega \in \Omega$. Therefore,

$$
d(u(\omega), v(\omega)) \leq H_{d}(F(\omega), G(\omega))+r(\omega), \quad \omega \in \Omega,
$$

which proves the result.

We are now ready to prove our random fixed point result.

Theorem 7.8. Let $(\Omega, \Sigma)$ be a measurable space, $X$ be a separable generalized Banach space, $C$ be a nonempty compact convex subset of $X$, and $G: \Omega \times C \rightarrow \mathscr{P}_{c p, c v}(C)$ be a random multivalued map that is continuous for every $\omega \in \Omega$ (i.e., is l.s.c. and u.s.c.). Then the operator inclusion $G$ has at least one random fixed point.

Proof. Let $\omega \in \Omega$. By Theorem 7.3, for a given $\varepsilon>0$, there exists a continuous map $f_{\varepsilon}: C \rightarrow X$ such that

$$
\mathscr{G} r\left(f_{\varepsilon}\right) \subset \mathscr{G} r(G(\omega, \cdot))+B(0, \varepsilon)
$$

and $f_{\varepsilon}(C) \subset \operatorname{co} G(\omega, C)$. Consider $G_{\varepsilon}: C \rightarrow C$ defined by $G_{\varepsilon}(y)=f_{\varepsilon}(x), x \in C$. By Theorem 7.4 , there exists $x_{\varepsilon}(\omega) \in C$ such that $x_{\varepsilon}(\omega)=f_{\varepsilon}\left(x_{\varepsilon}(\omega)\right)$. Let $\left\{\varepsilon_{n}: n \in \mathbb{N}\right\}$ be such that $\varepsilon_{n} \rightarrow 0$ as $n \rightarrow \infty$. Since $C$ is compact, there exists a subsequence $x_{\varepsilon_{n}}(\omega)$ in $C$ converging to $x(\omega) \in C$. So $f_{\varepsilon_{n}}\left(x_{\varepsilon_{n}}(\omega)\right) \rightarrow x(\omega)$, as $n \rightarrow \infty$. From (7.2), we get

$$
d\left(\left(x_{\varepsilon_{n}}(\omega), f_{\varepsilon_{n}}\left(x_{\varepsilon_{n}}(\omega)\right)\right), \mathscr{G} r(G(\omega, \cdot))\right) \leq \varepsilon_{n}, \text { for every } n \in \mathbb{N}
$$

It is clear that $G(\omega, \cdot)$ has a closed graph in $X \times X$ and consequently $x(\omega) \in G(\omega, x(\omega))$. Define $F_{*}: \Omega \rightarrow \mathscr{P}_{c l}(C)$ by $F_{*}(\omega)=\{x \in C: x \in G(\omega, x)\}$. By the continuity of $G(\omega, \cdot)$, for every 
$M \in \mathscr{P}_{c l}(C)$, we have

$$
G_{*}^{-1}(M)=\bigcap_{n=1}^{\infty} \bigcup_{x_{i} \in M_{n}}\left\{\omega \in C: d\left(x_{i}, F\left(\omega, x_{i}\right)\right)<\varepsilon_{n}\right\}
$$

where

$$
M_{n}=\left\{x \in C: d(x, M)<\frac{\varepsilon_{n}}{2}\right\}, \varepsilon_{n}:=\left(\begin{array}{c}
\frac{2}{n} \\
\vdots \\
\frac{2}{n}
\end{array}\right) .
$$

For every $i \in \mathbb{N}$, the multifunction $F\left(\cdot, x_{i}\right)$ is measurable. By the Castaing representation theorem (see [31] or [41, Theorem 2.75]), there exists a sequence of measurable functions $f_{p}: \Omega \rightarrow M, p \in \mathbb{N}$, such that $G\left(\omega, x_{i}\right)=\overline{\left\{f_{p}(\omega): p \in \mathbb{N}\right\}}$. Then,

$$
\left\{\omega \in C: d\left(x_{i}, G\left(\omega, x_{i}\right)\right)<\varepsilon_{n}\right\}=\bigcap_{p=1}^{\infty} \overline{\left\{\omega \in \Omega: d\left(x_{i}, f_{p}(\omega)\right)<\varepsilon_{n}\right\}} .
$$

It is clear that $g_{p}: \Omega \times C \rightarrow C$ defined by $g_{p}(\omega, x)=d\left(x, f_{p}(\omega)\right)$ is a Carathéodory function for each $p \in \mathbb{N}$. So the multifunction

$$
G_{p}(\omega)=\overline{\left\{\omega \in \Omega: d\left(x_{i}, f_{p}(\omega)\right)<\varepsilon_{n}\right\}},(\omega, x) \in \Omega \times C,
$$

is measurable. Hence $G_{*}^{-1}(M) \in \Sigma$. From Theorem 7.5, $G_{*}(\cdot)$ has at least one measurable selection that in turn is a random fixed point of $G$. This completes the proof.

\section{REFERENCES}

[1] O. Hanš, Random operator equations, Proceedings of 4th Berkeley Sympos. Math. Statist. Prob., vol. II, part I, pp. 185-202, University of California Press, Berkeley, 1961.

[2] A. Mukherjea, Random Transformations of Banach Spaces, Ph. D. Dissertation, Wayne State Univ., Detroit, Michigan, 1968.

[3] A. S̆paček, Zulfallige Gleichungen, Czechoslovak Math. J. 5 (1995), 462-466.

[4] A. T. Bharuch-Reid, Fixed point theorems in probabilistic analysis, Bull. Amer. Math. Soc. 82 (1976), 641657.

[5] B. L. S. Prakasa Rao, Stochastic integral equations of mixed type II, J. Math. Physical Sci. 7 (1973), 245-260.

[6] S. Itoh, A random fixed point theorem for a multivalued contraction mapping, Pacific J. Math. 68 (1977), 85-90.

[7] S. Itoh, S. Itoh, Nonlinear random equations with monotone operators in Banach spaces, Math. Ann. 236 (1978), 133-146.

[8] T. C. Lin, Random approximations and random fixed point theorems for non-self-maps, Proc. Amer. Math. Soc. 103 (1988), 1129-1135. 
[9] N. S. Papageorgiou, Random fixed point theorems for measurable multifunctions in Banach spaces, Proc. Amer. Math. Soc. 97 (1986), 507-514.

[10] V. M. Sehgal, S. P. Singh, On random approximations and a random fixed point theorem for set valued mappings, Proc. Amer. Math. Soc. 95 (1985), 91-94.

[11] T. N. Anh, Random equations and applications to general random fixed point theorems, New Zealand J. Math. $41(2011), 17-24$.

[12] H. D. Thang, P. T. Anh, Random fixed points of completely random operators, Random Oper. Stochastic Equations 21 (2013), 1-20.

[13] M. A. Krasnosel'skii, Some problems of nonlinear analysis, Amer. Math. Soc. Transl. 10 (1958), 345-409.

[14] J. Garcia-Falset, K. Latrach, E. Moreno-Gálvez, M. A Taoudi, Schaefer-Krasnosel'skii fixed points theorems using a usual measure of weak noncompactness, J. Differential Equations 352 (2012), 3436-3452.

[15] J. R. Graef, J. Henderson and A. Ouahab, Multivalued versions of a Krasnosel'skii type fixed point theorem, J. Fixed Point Theory Appl. 19 (2017), 1059-1082.

[16] A. Arunchai, S. Plubtieng, Random fixed point theorem of Krasnosel'skii type for the sum of two operators, Fixed Point Theory Appl. 2013 (2013), Article ID 142.

[17] J. Andres, L. Górniewicz, Topological Fixed Point Principles for Boundary Value Problems, Kluwer, Dordrecht, 2003.

[18] L. Górniewicz, Topological Fixed Point Theory of Multi-valued Mappings, Mathematics and its Applications, 495, Kluwer, Dordrecht, 1999.

[19] M. Kisielewicz, Differential Inclusions and Optimal Control, Kluwer, Dordrecht, 1991.

[20] K. Kuratowski, C. Ryll-Nardzewski, A general theorem on selectors, Bull. Acad. Polon. Sci. Ser. Sci. Math. Astronom. Phys. 13 (1965), 397-403.

[21] S. Hu, N. S. Papageorgiou, Handbook of Multivariate Analysis, Vol.I: Theory, Mathematics and its Applications, Vol. 419, Kluwer, Dordrecht, 1997.

[22] R. Kannan, Random correspondences and nonlinear equations, J. Multivariate Anal. 11 (1981), 230-243.

[23] E. Tarafdar, P. Watson, X. Z. Yuan, Jointly measurable selections of condensing Carathéodory set-valued mappings and its applications to random fixed points, Nonlinear Anal. 28 (1997), 39-48.

[24] T. Xiang, R. Yuan, A class of expansive-type Krasnosel'skii fixed point theorems, Nonlinear Anal. 71 (2009), 3229-3239.

[25] A. Cellina, A theorem on the approximation of compact set-valued mappings, Atti Accad. Naz. Lincei Rend. Cl. Sci. Fis. Mat. Natur. 47 (1969), 149-163.

[26] L. Górniewicz, A. Granas, W. Kryszewski, On the homotopy method in the fixed point index theory of multivalued mappings of compact absolute neighborhood retracts, J. Math. Anal. Appl. 161 (1991), 457-473.

[27] L. Górniewicz, M. Lassonde, Approximation and fixed points for compositions of $R_{\delta}$, Topology Appl. 55 (1994), 239-250.

[28] G. S. Ladde, V. Lakshmikantham, Random Differential Inequalities, Academic Press, New York, 1980. 
[29] V. Lupulescu, S. K. Ntouyas, Random fractional differential equations, Int. Electron. J. Pure Appl. Math. 4 (2012), 119-136.

[30] V. Lupulescu, D. O'Regan, G. Rahman, Existence results for random fractional differential equations, Opuscula Math. 34 (2014), 813-825.

[31] C. Castaing, M. Valadier, Convex Analysis and Measurable Multifunctions, Lecture Notes in Math., 580, Springer-Verlag, Berlin-Heidelberg-New York, 1977.

[32] D. Henry, Geometric Theory of Semilinear Parabolic Partial Differential Equations, Springer-Verlag, Berlin/New York, 1989.

[33] A. I. Perov, On the Cauchy problem for a system of ordinary differential equations, Priblijen. Metod Res. Dif. Urav Kiev, 1964 (in Russian).

[34] A. I. Perov, A. V. Kibenko, On a certain general method for investigation of boundary value problems, Izv. Akad. Nauk SSSR, Ser. Mat. 30 (1966), 249-264 (in Russian).

[35] A. Viorel, Contributions to the Study of Nonlinear Evolution Equations, Ph.D. thesis, Babeş-Bolyai University Cluj-Napoca Department of Mathematics, 2011.

[36] I. R. Petre, A. Petruşel, Krasnosels'kii's theorem in generalized Banach spaces and applications, Electron. J. Qual. Theory Differ. Equ 2012 (2012), Article ID 85.

[37] I. R. Petre, A multivalued version of Krasnosel'skii's theorem in generalized Banach spaces, An. Şt. Univ. “Ovidius" Constanţa Ser. Mat. 22 (2014), 177-192.

[38] M. L. Sinacer, J. J. Nieto, A. Ouahab, Random fixed point theorem in generalized Banach space and applications, Random Oper. Stochastic Equations 24 (2016), 93-112.

[39] I. A. Rus, The theory of a metrical fixed point theorem: theoretical and applicative relevances, Fixed Point Theory 9 (2008), 541-559.

[40] R. S. Varga, Matrix Iterative Analysis, 2nd revised and expanded, Springer Series in Computational Mathematics, Springer, Berlin, 2000.

[41] J. R. Graef, J. Henderson, A. Ouahab, Impulsive Differential Inclusions: A Fixed Point Approach, Walter de Gruyter GmbH, Berlin, 2013. 